\title{
Evolution of a Total Synthesis of (-)-Kendomycin Exploiting a Petasis-Ferrier Rearrangement/Ring-Closing Olefin Metathesis Strategy
}

\author{
SUPPORTING INFORMATION
}

Amos B. Smith, III, ${ }^{*}$ Eugen F. Mesaros, and Emmanuel A. Meyer

Department of Chemistry, Monell Chemical Senses Center, and Laboratory for Research on the Structure of Matter, University of Pennsylvania, Philadelphia, Pennsylvania 19104

E-mail: smithab@sas.upenn.edu 
Reactions were carried out in oven- or flame-dried glassware under an argon atmosphere, unless otherwise noted. All solvents were reagent grade. Diethyl ether $\left(\mathrm{Et}_{2} \mathrm{O}\right)$ and tetrahydrofuran (THF) were freshly distilled from sodium / benzophenone under argon. Reactions were magnetically stirred and monitored by thin layer chromatography (TLC) with $0.25 \mathrm{~mm} \mathrm{E}$. Merck pre-coated silica gel plates. Flash chromatography was performed with silica gel 60 (particle size $0.040-0.062 \mathrm{~mm}$ ) supplied by Silicycle and Sorbent Technologies. Yields refer to chromatographically and spectroscopically pure compounds, unless otherwise stated. All melting points were obtained on a Thomas-Hoover apparatus and are uncorrected. Infrared spectra were recorded on a Perkin-Elmer Model 283B spectrophotometer or a Jasco Model FT/IR-480 Plus spectrometer. Proton and carbon-13 NMR spectra were recorded on a Bruker AMX-500 spectrometer. Chemical shifts are reported relative to chloroform $(\delta 7.26)$, acetone $(\delta 2.04)$, or benzene $(\delta 7.15)$ for ${ }^{1} \mathrm{H}$ NMR and chloroform $(\delta 77.0)$, acetone $(\delta 29.8)$, or benzene $(\delta 128.0)$ for ${ }^{13} \mathrm{C}$ NMR. Optical rotations were measured on a Perkin-Elmer model 241 polarimeter. Highresolution mass spectra were measured at the University of Pennsylvania Mass Spectrometry Service Center on either a VG Micromass 70/70 H or VG ZAB-E spectrometer. X-ray intensity data were collected at the University of Pennsylvania on a Rigaku Mercury CCD area detector employing graphite-monochromated Mo-Ka radiation $(\lambda=0.71069 \AA)$ at a temperature of $143 \mathrm{~K}$. The X-ray crystallographic information files (CIF) for compounds (+)-30 and (-)-2 are provided.<smiles>COc1c(C)c(OC)c(OC(C)(C)C)c(C=O)c1Br</smiles>

Aldehyde 6: A solution of 3,5-dimethoxy-2-hydroxy-4-methyl-benzaldehyde ${ }^{1}(6.0 \mathrm{~g}, 30.5 \mathrm{mmol})$ in dichloromethane $(60 \mathrm{~mL})$ was treated with anhydrous potassium carbonate $(6.9 \mathrm{~g}, 50 \mathrm{mmol})$ and then cooled to $-10^{\circ} \mathrm{C}$. Bromine $(3.5 \mathrm{~mL}, 67.3 \mathrm{mmol})$ was added dropwise via addition funnel, then the reaction mixture was warmed to $0{ }^{\circ} \mathrm{C}$ and stirred for $1 \mathrm{~h}$ at this temperature. The reaction was quenched with a 1:1 (v/v) mixture of $10 \% \mathrm{Na}_{2} \mathrm{~S}_{2} \mathrm{O}_{3}$ and $10 \% \mathrm{NaHCO}_{3}$ aqueous solutions (60

\footnotetext{
${ }^{1}$ Kitahara, Y.; Nakahara, S.; Numata, R.; Kubo, A. Chem. Pharm. Bull. 1985, 33, 2122.
} 
$\mathrm{mL}$ ), warmed to room temperature, and then the aqueous phase was extracted with dichloromethane $(2 \times 50 \mathrm{~mL})$. The combined organic layers were washed with brine $(60 \mathrm{~mL})$, dried over $\mathrm{Na}_{2} \mathrm{SO}_{4}$, and then concentrated in vacuo. Flash chromatography purification (5\% ethyl acetate in hexanes) afforded the corresponding bromide ( $8.2 \mathrm{~g}, 97 \%$ yield) as a yellow solid: m.p. 95 - $96{ }^{\circ} \mathrm{C}$; IR ( $\left.\mathrm{CHCl}_{3}\right) 2998$ (w), 2940 (w), 2844 (w), 1631 (s), 1443 (m), 1401 (m), 1299 (s), 1268 (m), $1105(\mathrm{~m}), 998(\mathrm{~m}), 956(\mathrm{w}), 760(\mathrm{~m}) \mathrm{cm}^{-1} ;{ }^{1} \mathrm{H}$ NMR (500 MHz, $\left.\mathrm{CDCl}_{3}\right) \delta 12.07(\mathrm{~s}, 1 \mathrm{H}), 10.29$ (s, $1 \mathrm{H}), 3.87$ (s, $3 \mathrm{H}), 3.77(\mathrm{~s}, 3 \mathrm{H}), 2.31(\mathrm{~s}, 3 \mathrm{H}) ;{ }^{13} \mathrm{C}$ NMR $\left(125 \mathrm{MHz}, \mathrm{CDCl}_{3}\right) \delta$ 177.8, 154.2, $148.4,146.3,136.3,115.9,115.2,60.5,60.2,11.0$; high resolution mass spectrum $(\mathrm{Cl}+) \mathrm{m} / \mathrm{z}$ $273.9834\left[\mathrm{M}^{+}\right.$; calcd for $\mathrm{C}_{10} \mathrm{H}_{11} \mathrm{O}_{4} \mathrm{Br}$ : 273.9840].

A solution of the above bromide $(8.1 \mathrm{~g}, 29.0 \mathrm{mmol})$ in dimethylformamide $(23 \mathrm{~mL})$ was treated with diisopropylethylamine $(9.7 \mathrm{~mL}, 58.8 \mathrm{mmol})$ and $\mathrm{TBSCl}(8.9 \mathrm{~g}, 58.8 \mathrm{mmol})$ at room temperature. The reaction mixture was stirred for $1.5 \mathrm{~h}$, then quenched with water $(50 \mathrm{~mL})$. The aqueous phase was extracted with $\mathrm{Et}_{2} \mathrm{O}(3 \times 300 \mathrm{~mL})$, and the combined organic layers were washed with brine, dried over $\mathrm{Na}_{2} \mathrm{SO}_{4}$, and then concentrated in vacuo. Flash chromatography purification (2\% ethyl acetate in hexanes) afforded 6 (10.8 g, 94\% yield) as a yellow waxy solid: m.p. $34-36{ }^{\circ} \mathrm{C}$, IR $\left(\mathrm{CHCl}_{3}\right) 2933$ (s), 2858 (s), 1699 (s), 1448 (s), 1389 (s), 1250 (m), 1099 (m) $\mathrm{cm}^{-1} ;{ }^{1} \mathrm{H}$ NMR (500 MHz, $\left.\mathrm{CDCl}_{3}\right) \delta 10.42(\mathrm{~s}, 1 \mathrm{H}$ ), 3.39 (s, $3 \mathrm{H}$ ), $3.32(\mathrm{~s}, 3 \mathrm{H}), 2.07(\mathrm{~s}, 3 \mathrm{H}), 1.02$ (s, $9 \mathrm{H}), 0.14(\mathrm{~s}, 6 \mathrm{H}) ;{ }^{13} \mathrm{C}$ NMR $\left(125 \mathrm{MHz}, \mathrm{CDCl}_{3}\right) \delta 189.5,151.2,150.5,147.9,132.6,126.3$, $114.1,59.9,59.7,26.1,18.7,10.7,-4.2$; high resolution mass spectrum $(\mathrm{Cl}+) \mathrm{m} / \mathrm{z} 389.0783\left[\mathrm{M}^{+}\right.$; calcd for $\left.\mathrm{C}_{16} \mathrm{H}_{25} \mathrm{O}_{4} \mathrm{BrSi}: 389.0800\right]$.<smiles>C=C[C@H](C)CC[C@H](O)[C@H](C)C(=O)O</smiles>

$\beta$-Hydroxyacid (+)-7: Trifluoromethanesulfonic acid $(1.0 \mathrm{~mL}, 11.8 \mathrm{mmol})$ was added to a $1 \mathrm{M}$ solution of triethylborane $(11.6 \mathrm{~mL}, 11.6 \mathrm{mmol})$ in hexanes at $0{ }^{\circ} \mathrm{C}$. The reaction mixture was 
warmed to $40{ }^{\circ} \mathrm{C}$ for $30 \mathrm{~min}$ and then cooled back to $0{ }^{\circ} \mathrm{C}$. A pre-cooled $\left(0{ }^{\circ} \mathrm{C}\right)$ solution of oxazolidinone (+)-17 ${ }^{2}(2.6 \mathrm{~g}, 11.2 \mathrm{mmol})$ in dichloromethane $(30 \mathrm{~mL})$ was added dropwise, followed by diisopropylethylamine $(2.1 \mathrm{~mL}, 12.0 \mathrm{mmol})$, and after $30 \mathrm{~min}$ at $0{ }^{\circ} \mathrm{C}$, the reaction mixture was cooled to $-78^{\circ} \mathrm{C}$. Known aldehyde $(+)-16^{3}(1.23 \mathrm{~g}, 11.0 \mathrm{mmol})$ in dichloromethane (20 mL) was added dropwise to the reaction mixture, which was stirred at $-78{ }^{\circ} \mathrm{C}$ for $1 \mathrm{~h}$, and then at $0{ }^{\circ} \mathrm{C}$ for an additional $2 \mathrm{~h}$. The reaction was quenched at $0{ }^{\circ} \mathrm{C}$ with $\mathrm{pH} 7$ buffer $(18 \mathrm{~mL})$. After 10 min, a $1: 2(\mathrm{v} / \mathrm{v})$ mixture of $30 \% \mathrm{H}_{2} \mathrm{O}_{2}$ and methanol was added, and the mixture was stirred for $1 \mathrm{~h}$ at $0{ }^{\circ} \mathrm{C}$. Most of the solvent was evaporated in vacuo, and then the residue was extracted with EtOAc $(3 \times 150 \mathrm{~mL})$. The combined organic extracts were washed with 5\% aqueous $\mathrm{NaHCO}_{3}$ and brine (200 $\mathrm{mL}$ each), dried over $\mathrm{Na}_{2} \mathrm{SO}_{4}$, and then concentrated in vacuo. Flash chromatography purification with ethyl acetate in hexanes $(1: 20 \rightarrow 1: 4)$ as eluent afforded the corresponding aldol adduct $\left(3.2 \mathrm{~g}, 85 \%\right.$ yield) as a colorless oil: $[\alpha]_{\mathrm{D}}^{23}+61.1\left(c 0.825, \mathrm{CHCl}_{3}\right)$; IR ( $\left.\mathrm{CHCl}_{3}\right) 3526$ (br), 3065 (w), 2953 (s), 2865 (m), 1780 (s), 1684 (s), 1456 (w), 1387 (s), 1209 (s), $1110(\mathrm{~m}) \mathrm{cm}^{-1} ;{ }^{1} \mathrm{H}$ NMR (500 MHz, $\mathrm{CDCl}_{3}$ ) $\delta 7.33$ (dd, $\mathrm{J}=6.1,8.2 \mathrm{~Hz}, 2 \mathrm{H}$ ), 7.28 (dd, $\mathrm{J}=1.1$, $8.2 \mathrm{~Hz}, 1 \mathrm{H}$ ), 7.22 (dd, J = 1.1, $6.1 \mathrm{~Hz}, 2 \mathrm{H}$ ), 5.76 (ddd, $J=7.6,10.2,17.5 \mathrm{~Hz}, 1 \mathrm{H}$ ), 4.96 (ddd, $J=$ 1.2, 1.8, $17.5 \mathrm{~Hz}, 1 \mathrm{H}), 4.92$ (ddd, J = 0.8, 1.8, $10.2 \mathrm{~Hz}, 1 \mathrm{H}), 4.69(\mathrm{~m}, 1 \mathrm{H}), 4.23-4.18(\mathrm{~m}, 2 \mathrm{H})$, $3.94(\mathrm{~m}, 1 \mathrm{H}), 3.75(\mathrm{dq}, \mathrm{J}=2.6,7.1 \mathrm{~Hz}, 1 \mathrm{H}), 3.24(\mathrm{dd}, \mathrm{J}=3.3,13.3 \mathrm{~Hz}, 1 \mathrm{H}), 2.87(\mathrm{~d}, \mathrm{~J}=3.0 \mathrm{~Hz}$, $1 \mathrm{H}), 2.78(\mathrm{dd}, \mathrm{J}=9.4,13.4 \mathrm{~Hz}, 1 \mathrm{H}), 2.13(\mathrm{~m}, 1 \mathrm{H}), 1.60-1.45(\mathrm{~m}, 2 \mathrm{H}), 1.42-1.28(\mathrm{~m}, 2 \mathrm{H}), 1.25$ $(\mathrm{d}, \mathrm{J}=7.1 \mathrm{~Hz}, 3 \mathrm{H}), 1.00(\mathrm{~d}, \mathrm{~J}=6.7 \mathrm{~Hz}, 3 \mathrm{H}) ;{ }^{13} \mathrm{C} \mathrm{NMR}\left(125 \mathrm{MHz}, \mathrm{CDCl}_{3}\right) \delta 177.5,152.9,144.2$, $134.9,129.4,128.9,127.5,113.5,71.4,66.1,55.1,42.0,37.7,37.6,32.7,31.4,20.2,10.3 ;$ high resolution mass spectrum (ES+) $\mathrm{m} / \mathrm{z} 368.1822\left[(\mathrm{M}+\mathrm{Na})^{+}\right.$; calcd for $\mathrm{C}_{20} \mathrm{H}_{27} \mathrm{O}_{4} \mathrm{NNa}$ : 368.1837].

A solution of the above adduct $(3.0 \mathrm{~g}, 8.7 \mathrm{mmol})$ in a 5:1 (v/v) mixture of THF and water $(20 \mathrm{~mL})$ was treated with $30 \% \mathrm{H}_{2} \mathrm{O}_{2}(5.2 \mathrm{~mL})$ and $\mathrm{LiOH}(0.8 \mathrm{~g}, 19.1 \mathrm{mmol})$ at $0{ }^{\circ} \mathrm{C}$. After stirring the reaction mixture for $1 \mathrm{~h}$, a saturated aqueous $\mathrm{Na}_{2} \mathrm{SO}_{3}$ solution $(17 \mathrm{~mL})$ was slowly added, and the reaction was allowed to reach room temperature. The mixture was extracted with

\footnotetext{
${ }^{2}$ Evans, D. A.; Bartroli, J.; Shih, T. L. J. Am. Chem. Soc. 1981, 103, 2127.

3 Ireland, R. E.; Anderson, R. C.; Badoud, R.; Fitzsimmons, B. J.; McGarvey, G. J.; Thaisrivongs, S.; Wilcox, C. S. J. Am. Chem. Soc. 1983, 105, 1988. Barrett, A. G. M.; Carr, R. A. E.; Attwood, S. V.; Richardson, G.; Walshe, N. D. A. J. Org. Chem. 1986, 51, 4840.
} 
dichloromethane $(3 \times 60 \mathrm{~mL})$. The aqueous residue was diluted with water $(40 \mathrm{~mL})$, cooled to 0 ${ }^{\circ} \mathrm{C}$, neutralized with $3 \mathrm{~N}$ aqueous $\mathrm{HCl}$ to $\mathrm{pH} 2-3$, and then extracted with EtOAc $(3 \times 100 \mathrm{~mL})$. The combined organic extracts were washed with brine $(200 \mathrm{~mL})$, dried over $\mathrm{Na}_{2} \mathrm{SO}_{4}$, and then concentrated in vacuo. Flash chromatography purification with ethyl acetate in hexanes (1:1 $\rightarrow$ $100 \%)$ as eluent afforded acid (+)-7 (1.5 g, 92\% yield) as a colorless oil: $[\alpha]_{\mathrm{D}}^{23}+10.5$ (c 1.425, $\left.\mathrm{C}_{6} \mathrm{H}_{6}\right)$; IR $\left(\mathrm{CHCl}_{3}\right) 2958$ (br), 1716 (s), 1456 (w), 1253 (s), 1199 (m), 845 (s) cm ${ }^{-1} ;{ }^{1} \mathrm{H}$ NMR (500 $\mathrm{MHz}, \mathrm{CDCl}_{3}$ ) $\delta 5.66$ (ddd, $J=7.6,10.2,17.4 \mathrm{~Hz}, 1 \mathrm{H}$ ), 4.96 (apparent td, $J=1.0,17.5 \mathrm{~Hz}, 1 \mathrm{H}$ ), 4.93 (apparent td, $J=1.0,10.2 \mathrm{~Hz}, 1 \mathrm{H}), 3.93$ (m, $1 \mathrm{H}$ ), 2.58 (dq, $J=3.4,7.1 \mathrm{~Hz}, 1 \mathrm{H}$ ), 2.14 (apparent septet, $\mathrm{J}=6.7 \mathrm{~Hz}, 1 \mathrm{H}), 1.58-1.23(\mathrm{~m}, 4 \mathrm{H}), 1.20(\mathrm{~d}, \mathrm{~J}=7.2 \mathrm{~Hz}, 3 \mathrm{H}), 1.00(\mathrm{~d}, \mathrm{~J}=6.7$ $\mathrm{Hz}, 3 \mathrm{H}) ;{ }^{13} \mathrm{C}$ NMR $\left(125 \mathrm{MHz}, \mathrm{CDCl}_{3}\right) \delta 181.2,144.1,113.1,71.6,44.1,37.6,32.7,31.2,20.3$, 10.3; high resolution mass spectrum $(\mathrm{Cl}+) \mathrm{m} / \mathrm{z} 187.1329\left[(\mathrm{M}+\mathrm{H})^{+}\right.$; calcd for $\mathrm{C}_{10} \mathrm{H}_{19} \mathrm{O}_{3}$ : 187.1328].<smiles>C=C(C)C[C@H](C)CO</smiles>

Alcohol (+)-19: ${ }^{4}$-BuLi (2.5M in hexanes, $\left.38 \mathrm{~mL}, 95.4 \mathrm{mmol}\right)$ was added to diisopropylamine (13.4 $\mathrm{mL}, 95.4 \mathrm{mmol})$ in THF $(100 \mathrm{~mL})$ at $-78{ }^{\circ} \mathrm{C}$, and the mixture was stirred for $1 \mathrm{~h}$ at the same temperature. A solution of the Evans oxazolidinone $(+)-17(20.8 \mathrm{~g}, 89.2 \mathrm{mmol})$ in THF $(40 \mathrm{~mL})$ was then added dropwise via cannula at $-78{ }^{\circ} \mathrm{C}$, and the mixture was stirred for $30 \mathrm{~min}$. Methallyliodide (34 g, $180 \mathrm{mmol}$ ) was added slowly, and the reaction mixture was stirred for $1 \mathrm{~h}$ at $-78^{\circ} \mathrm{C}, 2 \mathrm{~h}$ at $-40^{\circ} \mathrm{C}$, and finally was allowed to warm to $-15^{\circ} \mathrm{C}$. The mixture was quenched by addition of saturated aqueous $\mathrm{NH}_{4} \mathrm{Cl}$, and the organic solvent was removed in vacuo. The residue was diluted with water and extracted with dichloromethane $(4 \times 100 \mathrm{~mL})$. The combined organic layers were dried over $\mathrm{MgSO}_{4}$ and concentrated in vacuo. Flash chromatography with 15 $\%$ ethyl acetate in hexanes provided the corresponding alkylation product ( $21.1 \mathrm{~g}, 82 \%$ yield) as a yellow oil: $[\alpha]_{D}^{23}+60.2\left(c\right.$ 1.65, $\mathrm{CH}_{2} \mathrm{Cl}_{2}$ ); IR (film) $3338(w), 3074(w), 2974(w), 2935$ (w), $1780(\mathrm{~s})$, $1696(\mathrm{~m}), 1455$ (w), $1387(\mathrm{~m}), 1349(\mathrm{~m}), 1237(\mathrm{~m}), 1208(\mathrm{~m}) ;{ }^{1} \mathrm{H}$ NMR $\left(500 \mathrm{MHz}, \mathrm{CDCl}_{3}\right) \delta 7.33$ (t, J = 7.2 Hz, 2 H), 7.27 (t, J = 7.2 Hz, 1 H), 7.21 (d, J = 6.9 Hz, 2 H), 4.81 (br s, 1 H), 4.76 (br s,

\footnotetext{
${ }^{4}$ Lit. value for the known enantiomer: $[\alpha]_{\mathrm{D}}^{23}-3.9\left(c\right.$ 4.1, $\left.\mathrm{CH}_{2} \mathrm{Cl}_{2}\right)$. See: Organ, M. G.; Bilokin, Y. V.; Bratovanov, S. J. Org. Chem. 2002, 67, 5176.
} 
$1 \mathrm{H}), 4.68$ (m, $1 \mathrm{H}), 4.21-4.14(\mathrm{~m}, 2 \mathrm{H}), 4.03(\mathrm{~m}, 1 \mathrm{H})$; 3.28 (dd, J = 3.3, $13.4 \mathrm{~Hz}, 1 \mathrm{H}), 2.71$ (dd, J = 9.7, 13.4 Hz, $1 \mathrm{H}), 2.56$ (dd, J = 7.0, $13.8 \mathrm{~Hz}, 1 \mathrm{H}$ ), 2.10 (dd, J = 7.5, $13.8 \mathrm{~Hz}, 1 \mathrm{H}), 1.78$ (s, 3 H), $1.16(\mathrm{~d}, \mathrm{~J}=6.8 \mathrm{~Hz}, 3 \mathrm{H}) ;{ }^{13} \mathrm{C}$ NMR $\left(125 \mathrm{MHz}, \mathrm{CDCl}_{3}\right) \delta 177.0,153.1,142.9,135.4,129.4$, $128.9,127.3,112.5,66.0,55.4,41.8,38.0,35.6,22.3,16.7$; high resolution mass spectrum $(\mathrm{Cl}+)$ $\mathrm{m} / \mathrm{z} 287.1515\left[\mathrm{M}^{+}\right.$; calcd for $\left.\mathrm{C}_{17} \mathrm{H}_{21} \mathrm{NO}_{3}: 287.1521\right]$.

$\mathrm{MeOH}(3.25 \mathrm{~mL}, 80.1 \mathrm{mmol})$ was added to a solution of the above material $(20.0 \mathrm{~g}, 69.7 \mathrm{mmol})$ in $\mathrm{Et}_{2} \mathrm{O}(300 \mathrm{~mL})$ at $0{ }^{\circ} \mathrm{C}$, followed by $\mathrm{LiBH}_{4}(1.838 \mathrm{~g}, 80.1 \mathrm{mmol})$ in two portions. The reaction mixture was maintained at $0{ }^{\circ} \mathrm{C}$ for $45 \mathrm{~min}$ and at room temperature for $90 \mathrm{~min}$. An aqueous solution of $\mathrm{NaOH}\left(11.2 \mathrm{~g}\right.$ in $125 \mathrm{~mL} \mathrm{H} \mathrm{H}_{2} \mathrm{O}$ ) was added, and the mixture was stirred until both phases were clear. The aqueous phase was further extracted with $\mathrm{Et}_{2} \mathrm{O}(3 \times 100 \mathrm{~mL})$ and the combined organic layers were washed with brine and dried over $\mathrm{MgSO}_{4}$. Flash chromatography (pentane/Et $\left.{ }_{2} \mathrm{O} 2: 1\right)$ provided (+)-19 $\left(7.32 \mathrm{~g}, 92 \%\right.$ yield) as a colorless liquid: $[\alpha]_{\mathrm{D}}^{23}+5.5(c 0.86$, $\left.\mathrm{CH}_{2} \mathrm{Cl}_{2}\right),{ }^{4}{ }^{1} \mathrm{H}$ NMR $\left(500 \mathrm{MHz}, \mathrm{CDCl}_{3}\right) \delta 4.77(\mathrm{~m}, 1 \mathrm{H}), 4.71(\mathrm{~m}, 1 \mathrm{H})$, 3.54-3.43 (m, $\left.2 \mathrm{H}\right), 2.14-2.12$ (m, $1 \mathrm{H}), 1.84-1.89(\mathrm{~m}, 2 \mathrm{H}), 1.73(\mathrm{~s}, 3 \mathrm{H}), 1.39(\mathrm{~m}, 1 \mathrm{H}), 0.90(\mathrm{~d}, \mathrm{~J}=6.5 \mathrm{~Hz}, 3 \mathrm{H})$.<smiles>C=C(C)C[C@H](C)C[C@H](C)C(=O)N(C)[C@@H](C)[C@H](O)c1ccccc1</smiles>

Alkylation product (-)-21: To a solution of alcohol (+)-19 (2.9 g, $25 \mathrm{mmol})$ in $\mathrm{Et}_{2} \mathrm{O} / \mathrm{CH}_{3} \mathrm{CN}(150$ $\mathrm{mL}, 1: 4)$ at $0{ }^{\circ} \mathrm{C}$ was added $\mathrm{PPh}_{3}(9.6 \mathrm{~g}, 36.8 \mathrm{mmol})$, imidazole $(4.5 \mathrm{~g}, 71.1 \mathrm{mmol})$, and $\mathrm{I}_{2}(9.0 \mathrm{~g}$, $35.5 \mathrm{mmol}$ ) portionwise. The reaction mixture was stirred for $3 \mathrm{~h}$ at room temperature and then treated with pentane $(300 \mathrm{~mL})$. The mixture was filtered, the precipitate washed with pentane, and the filtrate concentrated in vacuo $\left(0^{\circ} \mathrm{C}\right)$. Flash chromatography (pentane) provided the corresponding iodide $(4.7 \mathrm{~g}, 85 \%$ yield $)$ as a colorless liquid: $[\alpha]_{\mathrm{D}}^{23}-10.7$ (c 1.060, $\left.\mathrm{CHCl}_{3}\right)$; IR (film) 3073 (m), 2962 (s), 2924 (s), 1648 (m), 1452 (m), 1375 (m), 1194 (m), 892 (s); ${ }^{1} \mathrm{H}$ NMR $\left(500 \mathrm{MHz}, \mathrm{CDCl}_{3}\right) \delta 4.80$ (br s, $\left.1 \mathrm{H}\right), 4.74$ (br s, $\left.1 \mathrm{H}\right), 3.25$ (dd, J = 6.1, $\left.9.6 \mathrm{~Hz}, 1 \mathrm{H}\right), 3.12$ (dd, $J=$ 6.1, $9.6 \mathrm{~Hz}, 1 \mathrm{H}$ ), 2.10 (dd, J = 7.1, $13.7 \mathrm{~Hz}, 1 \mathrm{H}$ ), 1.89 (dd, J = 7.1, $13.7 \mathrm{~Hz}, 1 \mathrm{H}), 1.70(\mathrm{~s}, 3 \mathrm{H})$, $1.66(\mathrm{~m}, 1 \mathrm{H}), 0.98(\mathrm{~d}, \mathrm{~J}=6.5 \mathrm{~Hz}, 3 \mathrm{H}) ;{ }^{13} \mathrm{C} \operatorname{NMR}\left(125 \mathrm{MHz}, \mathrm{CDCl}_{3}\right) \delta 143.2,112.5,44.9,32.5$, 
27.2, 22.6, 17.2; high resolution mass spectrum $(\mathrm{Cl}+) \mathrm{m} / \mathrm{z} 224.0052\left[\mathrm{M}^{+}\right.$; calcd for $\mathrm{C}_{7} \mathrm{H}_{13} \mathrm{l}$ : 224.0826].

$n$-BuLi (2.5M in hexanes, $32 \mathrm{~mL}, 80 \mathrm{mmol})$ was added to a suspension of $\mathrm{LiCl}(10.7 \mathrm{~g}, 254.0$ $\mathrm{mmol}$, dried for $18 \mathrm{~h}$ at $130{ }^{\circ} \mathrm{C}$ under high vacuum) and diisopropylamine $(12.0 \mathrm{~mL}, 86.0 \mathrm{mmol})$ in THF $(55 \mathrm{~mL})$ at $-78{ }^{\circ} \mathrm{C}$. The resulting suspension was warmed to $0{ }^{\circ} \mathrm{C}$ for $10 \mathrm{~min}$. An ice-cooled solution of $(R, R)$-pseudoephedrine propionamide ${ }^{5} 20(9.3 \mathrm{~g}, 42.0 \mathrm{mmol})$ in THF $(120 \mathrm{~mL})$ was added at $-78^{\circ} \mathrm{C}$ and the mixture was stirred for $1 \mathrm{~h}$ at $-78^{\circ} \mathrm{C}, 15 \mathrm{~min}$ at $0{ }^{\circ} \mathrm{C}$, and $5 \mathrm{~min}$ at room temperature. To this solution was added the iodide described above $(4.5 \mathrm{~g}, 20.0 \mathrm{mmol})$ at $0{ }^{\circ} \mathrm{C}$, and the reaction mixture was stirred at room temperature for $14 \mathrm{~h}$. The yellow reaction mixture was treated with half-saturated aqueous $\mathrm{NH}_{4} \mathrm{Cl}(100 \mathrm{~mL})$ and extracted with EtOAc $(4 \times 100 \mathrm{~mL})$. The combined organic layers were dried over $\mathrm{Na}_{2} \mathrm{SO}_{4}$ and concentrated in vacuo. Flash chromatography (hexanes/EtOAc 3:2) yielded (-)-21 (6.2 g, 98\% yield) as a yellow oil: $[\alpha]_{\mathrm{D}}^{23}-$ 81.6 (c 1.000, $\mathrm{C}_{6} \mathrm{H}_{6}$ ); IR (film) 3384 (s), 3069 (w), 3029 (w), 2965 (m), 2929 (m), 1619 (s), 1453 (m), $1409(\mathrm{~m}), 1375(\mathrm{~m}) ;{ }^{1} \mathrm{H}$ NMR (4:1 rotamer ratio, the asterisk denotes the signals of the minor rotamer, $\left.500 \mathrm{MHz}, \mathrm{C}_{6} \mathrm{D}_{6}\right) \delta 7.34(\mathrm{~d}, \mathrm{~J}=7.7 \mathrm{~Hz}, 2 \mathrm{H}), 7.24^{*}(\mathrm{~d}, \mathrm{~J}=7.3 \mathrm{~Hz}, 2 \mathrm{H}), 6.98-7.19(\mathrm{~m}, 3$ H); 4.92 (br s, $1 \mathrm{H}), 4.86^{\star}(\mathrm{br} \mathrm{s}, 1 \mathrm{H}), 4.83^{\star}$ (br s, $\left.1 \mathrm{H}\right), 4.80$ (br s, $\left.1 \mathrm{H}\right), 4.73$ (br s, $\left.1 \mathrm{H}\right), 4.56$ (apparent t, J = 6.7 Hz, $1 \mathrm{H}), 4.36(\mathrm{br} \mathrm{s}, 1 \mathrm{H}), 4.23^{*}(\mathrm{~m}, 1 \mathrm{H}), 3.98^{\star}(\mathrm{m}, 1 \mathrm{H}), 3.01^{*}(\mathrm{~m}, 1 \mathrm{H}), 2.99^{*}$ (s, $3 \mathrm{H}), 2.44(\mathrm{~m}, 1 \mathrm{H}), 2.36(\mathrm{~s}, 3 \mathrm{H}), 1.99^{\star}(\mathrm{m}, 1 \mathrm{H}), 1.92-1.80(\mathrm{~m}, 1 \mathrm{H}), 1.70(\mathrm{~m}, 1 \mathrm{H}), 1.57$ (s, 3 H), $1.54(\mathrm{~m}, 1 \mathrm{H}), 1.27(\mathrm{~m}, 1 \mathrm{H}), 1.08^{*}(\mathrm{~d}, J=6.7 \mathrm{~Hz}, 3 \mathrm{H}), 1.00(\mathrm{~d}, J=6.7 \mathrm{~Hz}, 3 \mathrm{H}), 0.99(\mathrm{~d}, J=$ $7.0 \mathrm{~Hz}, 3 \mathrm{H}), 0.78(\mathrm{~d}, \mathrm{~J}=6.0 \mathrm{~Hz}, 3 \mathrm{H}), 0.68^{\star}(\mathrm{d}, \mathrm{J}=6.6 \mathrm{~Hz}, 3 \mathrm{H}) ;{ }^{13} \mathrm{C}$ NMR (the asterisk denotes the signals of the minor rotamer, $\left.125 \mathrm{MHz}, \mathrm{C}_{6} \mathrm{D}_{6}\right) \delta 178.3,171.1^{\star}, 144.7^{\star}, 143.7,142.7^{\star}, 128.7^{*}$, $128.3,127.4,127.3^{\star}, 126.8,112.1,112.0^{\star}, 76.4,75.3^{\star}, 59.0,58.2,46.5^{\star}, 46.4,41.5,34.4,33.5^{\star}$, $32.8^{\star}, 28.8,26.9^{\star}, 22.4^{\star}, 22.2,19.7,18.1^{\star}, 17.4,15.5^{\star}, 14.3$ (two overlapped signals); high resolution mass spectrum $(\mathrm{Cl}+) \mathrm{m} / \mathrm{z} 318.2444\left[(\mathrm{M}+\mathrm{H})^{+}\right.$; calcd for $\mathrm{C}_{20} \mathrm{H}_{32} \mathrm{NO}_{2}$ : 318.2433].

\footnotetext{
${ }^{5}$ Myers, A. G.; Yang, B. H.; Chen, H.; McKinstry, L.; Kopecky, D. J.; Gleason, J. L. J. Am. Chem. Soc. 1997, 119,6496 .
} 


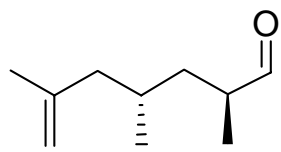

Aldehyde (+)-11. Method A: To a slurry of $\mathrm{LiAlH}_{4}(0.7 \mathrm{~g}, 18.1 \mathrm{mmol})$ in hexanes $(42 \mathrm{~mL}$, freshly distilled from $\left.\mathrm{CaH}_{2}\right)$ at $0{ }^{\circ} \mathrm{C}$ was added via syringe pump EtOAc $(2.6 \mathrm{~mL}$, freshly distilled from $\left.\mathrm{CaH}_{2}\right)$ over $1 \mathrm{~h}$. The mixture was stirred at $0{ }^{\circ} \mathrm{C}$ for $45 \mathrm{~min}$ and then $(-)-21(2.5 \mathrm{~g}, 7.9 \mathrm{mmol})$ in THF $(27 \mathrm{~mL})$ was added via cannula at $-78{ }^{\circ} \mathrm{C}$. The reaction mixture was stirred at $0{ }^{\circ} \mathrm{C}$ for 1.25 $\mathrm{h}$ and then transferred via pipette to ice-cold TFA $(6 \mathrm{~mL})$ in $1 \mathrm{M} \mathrm{HCl}(100 \mathrm{~mL})$. The mixture was stirred for $5 \mathrm{~min}$ at room temperature and then extracted with pentane- $\mathrm{Et}_{2} \mathrm{O}(9: 1,3 \times 100 \mathrm{~mL})$. The organic layers were basified with saturated aqueous $\mathrm{NaHCO}_{3}(50 \mathrm{~mL})$, and the separated aqueous phase was extracted with pentane- $\mathrm{Et}_{2} \mathrm{O}(9: 1,3 \times 50 \mathrm{~mL})$. The combined organic extracts were concentrated to one third of the volume in vacuo and filtered through a $\mathrm{SiO}_{2}$ pad (pentane/ $\left.\mathrm{Et}_{2} \mathrm{O} 9: 1\right)$. The solvent was removed in vacuo and flash chromatography $\left(1.5 \% \mathrm{Et}_{2} \mathrm{O}\right.$ in pentane as eluent) provided (+)-11 (960 mg, 77\% yield) as a colorless liquid.

Method B: $n$-BuLi (2.5M in hexanes, $65 \mathrm{mmol}$ ) was added to diisopropylamine (9.8 $\mathrm{ml}, 70 \mathrm{mmol})$ in THF $(70 \mathrm{~mL})$ at $-78{ }^{\circ} \mathrm{C}$. After stirring the mixture at $-78{ }^{\circ} \mathrm{C}$ for $10 \mathrm{~min}$ and at $0{ }^{\circ} \mathrm{C}$ for $10 \mathrm{~min}$, $\mathrm{BH}_{3}-\mathrm{NH}_{3}(2.3 \mathrm{~g}, 66.8 \mathrm{mmol})$ was added in one portion. After stirring the mixture at $0{ }^{\circ} \mathrm{C}$ for 15 min and then at room temperature for $15 \mathrm{~min},(-)-21(5.3 \mathrm{~g}, 16.7 \mathrm{mmol})$ in THF (45 mL) was added via cannula at $0{ }^{\circ} \mathrm{C}$ and the mixture was stirred at room temperature for $3 \mathrm{~h}$. The reaction was quenched by slow addition of aqueous $\mathrm{HCl}(3 \mathrm{~N}, 150 \mathrm{~mL})$ at $0{ }^{\circ} \mathrm{C}$ (gas evolution!). The aqueous phase was extracted with $\mathrm{Et}_{2} \mathrm{O}(4 \times 50 \mathrm{~mL})$, and the combined organic layers were washed with aqueous $\mathrm{HCl}(3 \mathrm{~N}, 25 \mathrm{~mL})$, aqueous $\mathrm{NaOH}(2 \mathrm{~N}, 25 \mathrm{~mL})$, and brine $(25 \mathrm{~mL})$, and dried over $\mathrm{Na}_{2} \mathrm{SO}_{4}$. After concentration in vacuo, purification by flash chromatography (pentane/Et ${ }_{2} \mathrm{O} 95: 5 \rightarrow$ 80:20) provided the $\mathrm{C}(19)$ alcohol $(2.5 \mathrm{~g}, 97 \%$ yield) as a colorless liquid: $[\alpha]_{\mathrm{D}}^{23}-31.8\left(\mathrm{c} 0.880, \mathrm{CHCl}_{3}\right)$; IR (film) 3334 (s), 2959 (s), 2918 (s), 2873 (s), 1649 (w), 1457 (m), 1377 (m), $1073(\mathrm{~m}), 807$ (m); ${ }^{1} \mathrm{H}$ NMR (500 MHz, $\mathrm{CDCl}_{3}$ ) $\delta 4.74$ (br s, $1 \mathrm{H}$ ), 4.66 (br s, $1 \mathrm{H}$ ), 3.44 (m, 2 H), 1.97 (dd, J = 6.8, 13.6 Hz, 1 H), 1.87 (dd, J = 7.7, 13.6, Hz, 1 H), 1.73 (m, 1 H), 1.69 (s, 3 H), 1.50 (br s, 1 H), $1.34(m, 1 \mathrm{H}), 1.11(\mathrm{~m}, 2 \mathrm{H}), 0.90(\mathrm{~d}, \mathrm{~J}=6.7 \mathrm{~Hz}, 3 \mathrm{H}), 0.84(\mathrm{~d}, \mathrm{~J}=6.5 \mathrm{~Hz}, 3$ 
H); ${ }^{13} \mathrm{C}$ NMR $\left(125 \mathrm{MHz}, \mathrm{CDCl}_{3}\right) \delta 144.6,111.5,69.1,52.1,40.3,33.3,27.7,22.2,19.3,16.2 ;$ high resolution mass spectrum $(\mathrm{Cl}+) \mathrm{m} / \mathrm{z} 156.1513\left[(\mathrm{M}+\mathrm{H})^{+}\right.$; calcd for $\left.\mathrm{C}_{10} \mathrm{H}_{21} \mathrm{O}: 156.1514\right]$.

To a solution of the above alcohol $(1.6 \mathrm{~g}, 10.2 \mathrm{mmol})$ in $\mathrm{CH}_{2} \mathrm{Cl}_{2} / \mathrm{DMSO}(1.5: 1,65 \mathrm{~mL})$ was added $\mathrm{Et}_{3} \mathrm{~N}(14.2 \mathrm{~mL}, 100 \mathrm{mmol})$ and $\mathrm{SO}_{3} \cdot \mathrm{Py}(6.5 \mathrm{~g}, 40.9 \mathrm{mmol})$ at $0{ }^{\circ} \mathrm{C}$. The reaction mixture was stirred at $0{ }^{\circ} \mathrm{C}$ for $1 \mathrm{~h}$ and quenched with saturated aqueous $\mathrm{NaHCO}_{3}(50 \mathrm{~mL})$. After extraction of the aqueous phase with $\mathrm{Et}_{2} \mathrm{O}(3 \times 50 \mathrm{~mL})$, the combined organic layers were washed with aqueous $\mathrm{NaHSO}_{4}(1 \mathrm{M}, 2 \times 50 \mathrm{~mL})$, saturated aqueous $\mathrm{NaHCO}_{3}(1 \times 100 \mathrm{~mL})$, brine $(2 \times 100 \mathrm{~mL})$, dried over $\mathrm{Na}_{2} \mathrm{SO}_{4}$, and concentrated in vacuo. Flash chromatography provided aldehyde (+)-11 (1.4 g, 89\%) as a colorless liquid: $[\alpha]_{\mathrm{D}}^{23}+6.3$ (c 1.240, $\mathrm{CHCl}_{3}$ ); IR (film) 3074 (w), 2967 (s), 2929 (s), 1707 (s), 1649 (w), 1458 (m), 1377 (m), 888 (m); ${ }^{1} \mathrm{H}$ NMR (500 MHz, CDCl $\left.)_{3}\right) \delta 9.61$ (d, J = 1.9 Hz, 1 H), 4.76 (br s, 1 H), 4.66 (br s, 1 H), 2.43 (m, 1 H), 2.00 (dd, J = 6.6, 13.5 Hz, 1 H) 1.90 (dd, $J=6.6,13.5 \mathrm{~Hz}, 1 \mathrm{H}), 1.70(\mathrm{~m}, 1 \mathrm{H}), 1.67(\mathrm{~s}, 3 \mathrm{H}), 1.48(\mathrm{~m}, 1 \mathrm{H}), 1.30(\mathrm{~m}, 1 \mathrm{H}), 1.08(\mathrm{~d}, \mathrm{~J}=7.0$ $\mathrm{Hz}, 3 \mathrm{H}), 0.87$ (d, J = 6.6 Hz, $3 \mathrm{H}) ;{ }^{13} \mathrm{C}$ NMR $\left(125 \mathrm{MHz}, \mathrm{CDCl}_{3}\right) \delta 205.2,144.0,112.0,46.3,44.3$, 37.3, 28.1, 22.1, 19.2, 13.3; high resolution mass spectrum $(\mathrm{Cl}+) \mathrm{m} / \mathrm{z} 153.1272\left[(\mathrm{M}-\mathrm{H})^{+}\right.$; calcd for $\mathrm{C}_{10} \mathrm{H}_{17} \mathrm{O}:$ 153.1280].

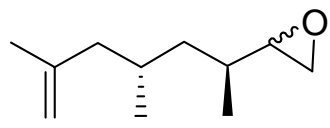

Epoxides 5. Method A: To a solution of aldehyde (+)-11 (100 mg, $0.65 \mathrm{mmol})$ and $\mathrm{CH}_{2} \mathrm{Br}_{2}(0.06$ $\mathrm{mL}, 0.85 \mathrm{mmol})$ in THF $(2 \mathrm{~mL})$ was added dropwise $1.6 \mathrm{M} n$-BuLi in hexanes $(0.49 \mathrm{~mL}, 0.78$ $\mathrm{mmol}$ ) at $-78{ }^{\circ} \mathrm{C}$. The reaction mixture was then stirred for $18 \mathrm{~h}$ at room temperature and quenched by addition of saturated aqueous $\mathrm{NH}_{4} \mathrm{Cl}(2 \mathrm{~mL})$. The aqueous phase was extracted with $\mathrm{CH}_{2} \mathrm{Cl}_{2}(3 \times 25 \mathrm{~mL})$, and the combined organic layers were dried over $\mathrm{Na}_{2} \mathrm{SO}_{4}$, and then concentrated in vacuo. Flash chromatography (hexanes/EtOAc 98:2) provided 5 (69 mg, 63\% yield, 1.8:1 mixture of diastereomers) as a colorless liquid: IR (film) 3073 (w), 3047 (w), 2965 (s), 2819 (s), $1649(\mathrm{~m}), 1458(\mathrm{~m}), 1376(\mathrm{~m}), 887(\mathrm{~m}) ;{ }^{1} \mathrm{H}$ NMR (The asterisk denotes the minor diastereomer, $500 \mathrm{MHz}, \mathrm{CDCl}_{3}$ ), 4.74 (br s, $1 \mathrm{H}$ ), 4.65 (br s, $\left.1 \mathrm{H}\right)$, 2.66-2.75 (m, $\left.2 \mathrm{H}\right), 2.53$ (dd, J $=2.8,4.9 \mathrm{~Hz}, 1 \mathrm{H}), 2.49^{*}(\mathrm{dd}, \mathrm{J}=2.9,4.8 \mathrm{~Hz}, 1 \mathrm{H}), 1.73-2.04(\mathrm{~m}, 3 \mathrm{H}), 1.73^{\star}(\mathrm{s}, 3 \mathrm{H}), 1.72(\mathrm{~s}, 3$ 
H), 1.12-1.44 (m, $3 \mathrm{H}), 1.01(\mathrm{~d}, J=6.6 \mathrm{~Hz}, 3 \mathrm{H}), 0.91^{*}(\mathrm{~d}, \mathrm{~J}=6.9 \mathrm{~Hz}, 3 \mathrm{H}), 0.83^{\star}(\mathrm{d}, J=6.5 \mathrm{~Hz}, 3$ $\mathrm{H}$ ), $0.82\left(\mathrm{~d}, \mathrm{~J}=6.5 \mathrm{~Hz}, 3 \mathrm{H}\right.$ ); ${ }^{13} \mathrm{C}$ NMR (The asterisk denotes the minor diastereomer, $125 \mathrm{MHz}$, $\left.\mathrm{CDCl}_{3}\right) \delta 144.7^{\star}, 144.5,111.8,111.7^{\star}, 57.6,57.5^{\star}, 47.0,46.7^{\star}, 46.6,46.0^{\star}, 41.6^{\star}, 40.4,33.9$ $33.7^{\star}, 27.7,22.3,19.5,19.4^{\star}, 15.4,14.2^{*}$; high resolution mass spectrum $(\mathrm{Cl}+) \mathrm{m} / \mathrm{z} 169.1583$ $\left[(\mathrm{M}+\mathrm{H})^{+}\right.$; calcd for $\left.\mathrm{C}_{11} \mathrm{H}_{21} \mathrm{O}: 169.1592\right]$.

Method B: To NaH (19.4 g, $0.49 \mathrm{mmol}, 60 \%$ dispersion in mineral oil, washed twice with anhydrous hexane) was added DMSO $(0.8 \mathrm{~mL})$ at room temperature, and the mixture was stirred for $1 \mathrm{~h}$. To the resulting suspension was added (R)-(dimethylamino)methylphenyloxosulfoxonium fluoroborate (133 mg, $0.49 \mathrm{mmol}$ ) in one portion (gas evolution!). The solution was stirred for 45 min at room temperature. A solution of $(+)-11(50 \mathrm{mg}, 0.32 \mathrm{mmol})$ in DMSO (1.3 mL) was added via cannula at room temperature and the reaction mixture was stirred for $12 \mathrm{~h}$. After addition of water $(1 \mathrm{~mL})$, the aqueous phase was extracted with $\mathrm{Et}_{2} \mathrm{O}(4 \times 10 \mathrm{~mL})$. The combined organic layers were washed with brine $(2 \times 15 \mathrm{~mL})$, dried over $\mathrm{Na}_{2} \mathrm{SO}_{4}$ and concentrated in vacuo. The crude mixture was dissolved in $\mathrm{EtOH}(5 \mathrm{~mL})$, treated with $\mathrm{NaBH}_{4}(9.8 \mathrm{mg})$ at $0{ }^{\circ} \mathrm{C}$ for $30 \mathrm{~min}$ and then at room temperature for $15 \mathrm{~min}$ and subjected to the same work-up as described in method A. Preparative thin-layer chromatography (pentane/ $\mathrm{Et}_{2} \mathrm{O}$ 9:1) provided epoxide 5 (34 mg, 62\% yield) in a diastereomeric ratio of 3.7:1.

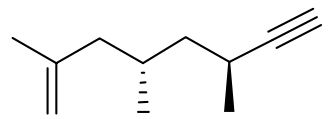

Alkyne (+)-10: To a stirred solution of KOt-Bu (102 mg, $0.74 \mathrm{mmol})$ in THF $(2 \mathrm{~mL})$ was added dropwise dimethyl-diazomethylphosphonate ${ }^{6}(111 \mathrm{mg}, 0.74 \mathrm{mmol})$ in THF $(2 \mathrm{~mL})$ at $-78{ }^{\circ} \mathrm{C}$. After stirring the reaction mixture at $-78{ }^{\circ} \mathrm{C}$ for $15 \mathrm{~min}$, aldehyde (+)-11 (100 mg, $\left.0.65 \mathrm{mmol}\right)$ in THF (3 $\mathrm{mL}$ ) was added via cannula over $15 \mathrm{~min}$ (gas evolution!). After $1 \mathrm{~h}$ at $-78{ }^{\circ} \mathrm{C}$, the reaction was quenched by addition of saturated aqueous $\mathrm{NH}_{4} \mathrm{Cl}(2 \mathrm{~mL})$, and the aqueous layer was extracted with pentane $(3 \times 15 \mathrm{~mL})$. The combined organic layers were dried over $\mathrm{Na}_{2} \mathrm{SO}_{4}$ and

\footnotetext{
${ }^{6}$ (a) Seyferth, D.; Marmor, R. S.; Hilbert, P. J. Org. Chem. 1971, 36, 1379. (b) Okada, N.; Nishimura, S. J. Biol. Chem. 1979, 254, 3061. (c) Gilbert, J. C.; Weerasooriya, U. J. Org. Chem. 1982, 47, 1837. (d) Ohira, S. Synth. Comm. 1989, 19, 561.
} 
concentrated in vacuo $\left(0^{\circ} \mathrm{C}\right)$. Flash chromatography (pentane) provided $(+)-10(75 \mathrm{mg}, 77 \%$ yield) as a colorless liquid: $[\alpha]_{\mathrm{D}}^{23}+17.8$ (c 1.100, $\mathrm{CHCl}_{3}$ ); IR (film) 3311 (s), 2969 (s), 2922 (s), 1652 (m), 1456 (m), $1376(\mathrm{~m}) ;{ }^{1} \mathrm{H}$ NMR (500 MHz, $\mathrm{CDCl}_{3}$ ) $\delta 4.74$ (br s, $1 \mathrm{H}$ ), 4.66 (br s, $\left.1 \mathrm{H}\right), 2.53$ (m, $1 \mathrm{H}), 2.00(\mathrm{dd}, J=5.2,13.1 \mathrm{~Hz}, 1 \mathrm{H}), 2.03(\mathrm{~d}, \mathrm{~J}=2.5,6.6 \mathrm{~Hz}, 1 \mathrm{H}), 1.84(\mathrm{~m}, 1 \mathrm{H}), 1.75(\mathrm{dd}, \mathrm{J}=$ 9.0, 13.1 Hz, 1H), $1.70(\mathrm{~s}, 3 \mathrm{H}), 1.35(\mathrm{~m}, 2 \mathrm{H}), 1.17(\mathrm{~d}, \mathrm{~J}=6.9 \mathrm{~Hz}, 3 \mathrm{H}), 0.86(\mathrm{~d}, \mathrm{~J}=6.5 \mathrm{~Hz}, 3 \mathrm{H})$; ${ }^{13} \mathrm{C}$ NMR $\left(125 \mathrm{MHz}, \mathrm{CDCl}_{3}\right) \delta 144.6,111.6,89.4,68.0,45.4,44.2,28.6,23.4,22.1,20.9,19.8$; high resolution mass spectrum $(\mathrm{Cl}+) \mathrm{m} / \mathrm{z} 135.1157\left[(\mathrm{M}-\mathrm{Me})^{+}\right.$; calcd for $\mathrm{C}_{10} \mathrm{H}_{15}$ : 135.1174].

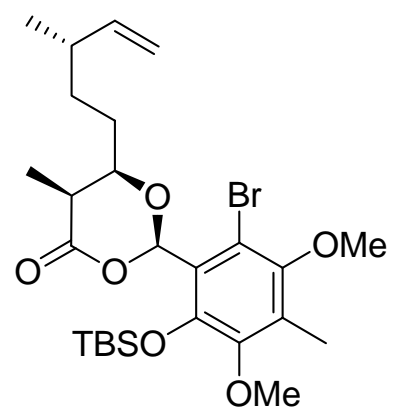

Dioxanone (+)-23: To a solution of aldehyde $6(2.5 \mathrm{~g}, 6.4 \mathrm{mmol})$ and acid (+)-7 (1.0 g, $5.4 \mathrm{mmol})$ in dichloromethane $(55 \mathrm{~mL})$ was added $i$-PrOTMS $(3.8 \mathrm{~mL}, 21.5 \mathrm{mmol})^{7}$ and TMSOTf $(0.4 \mathrm{~mL}$, $2.1 \mathrm{mmol}$ ) at $-78^{\circ} \mathrm{C}$, and the reaction mixture was stirred overnight at the same temperature. The reaction was quenched at $-78{ }^{\circ} \mathrm{C}$ with pyridine $(5 \mathrm{~mL})$, and then concentrated in vacuo. Flash chromatography through silylated silica gel, with ethyl acetate in hexanes $(1: 19 \rightarrow 1: 1)$ as eluent, afforded dioxanone $(+)-23(2.3 \mathrm{~g}, 77 \%$ yield $)$ as a pale yellow oil: $[\alpha]_{\mathrm{D}}^{23}+4.5\left(c \quad 0.600, \mathrm{C}_{6} \mathrm{H}_{6}\right)$; IR $\left(\mathrm{CHCl}_{3}\right) 2932$ (s), 1751 (s), 1456 (s), 1399 (s), 1332 (w), 1245 (s), 999 (m), 840 (s) cm ${ }^{-1} ;{ }^{1} \mathrm{H}$ NMR (500 MHz, $\left.\mathrm{C}_{6} \mathrm{D}_{6}\right) \delta 6.99(\mathrm{~s}, 1 \mathrm{H}$ ), 5.50 (ddd, $J=7.7,10.5,17.0 \mathrm{~Hz}, 1 \mathrm{H}$ ), 4.89 (ddd, $J=1.2,1.8$, $17.0 \mathrm{~Hz}, 1 \mathrm{H}$ ), 4.88 (ddd, J = 0.7, 1.0, $10.5 \mathrm{~Hz}, 1 \mathrm{H}$ ), 3.84 (td, J = 4.6, 9.2 Hz, $1 \mathrm{H}$ ), 3.40 (s, $3 \mathrm{H}$ ), 3.21 (s, 3 H), 2.56 (m, 1 H), 2.07 (s, 3 H), 1.91 (apparent septet, J = 7.1 Hz, 1 H), $1.62(\mathrm{~m}, 1 \mathrm{H}$ ), $1.36(\mathrm{~d}, \mathrm{~J}=7.4 \mathrm{~Hz}, 3 \mathrm{H}), 1.36(\mathrm{~m}, 1 \mathrm{H}), 1.23(\mathrm{~m}, 1 \mathrm{H}), 1.10(\mathrm{~m}, 1 \mathrm{H}), 1.03(\mathrm{~s}, 9 \mathrm{H}), 0.86(\mathrm{~d}, \mathrm{~J}=6.7$ $\mathrm{Hz}, 3 \mathrm{H}), 0.20$ (s, $3 \mathrm{H}), 0.16(\mathrm{~s}, 3 \mathrm{H}) ;{ }^{13} \mathrm{C}$ NMR $\left(125 \mathrm{MHz}, \mathrm{C}_{6} \mathrm{D}_{6}\right) \delta 170.8,151.9,149.6,145.2$, 144.1, 129.1, 125.0, 113.4, 113.2, 97.9, 78.8, 59.8, 59.7, 39.7, 37.9, 32.2, 29.8, 26.2, 20.2, 18.7,

\footnotetext{
${ }^{7}$ Kurihara, M.; Hakamata, W. J. Org. Chem. 2003, 68, 3413.
} 
12.1, 10.4, -4.3, -4.5; high resolution mass spectrum $(\mathrm{ES}+) \mathrm{m} / \mathrm{z} 579.1731\left[(\mathrm{M}+\mathrm{Na})^{+}\right.$; calcd for $\mathrm{C}_{26} \mathrm{H}_{41} \mathrm{O}_{6} \mathrm{BrSiNa}$ : 579.1753].<smiles>C=C[C@H](C)CCC1O[C@@H](c2c(Br)c(OC)c(C)c(OC)c2OC(F)(F)C(C)(C)C)OC(=C)[C@@H]1C</smiles>

Enol-acetal 24: Freshly prepared $\mathrm{Cp}_{2} \mathrm{TiMe}_{2}(3.3 \mathrm{~g}, 16.0 \mathrm{mmol})$ in THF (32 mL) was added to a solution of $(+)-23(3.6 \mathrm{~g}, 6.4 \mathrm{mmol})$ in THF $(13 \mathrm{~mL})$, and then the reaction mixture was stirred at $60-63{ }^{\circ} \mathrm{C}$ (external temperature) for $24 \mathrm{~h}$, in the dark. After cooling to room temperature, the reaction mixture was diluted with hexanes $(250 \mathrm{~mL})$ and the resulting slurry was filtered through Celite to remove the titanium salts. The filtrate was concentrated in vacuo and purified by flash chromatography through silylated silica gel, with $5 \%$ ethyl acetate in hexanes as eluent to afford enol-acetal 24 ( $3.0 \mathrm{~g}, 85 \%$ yield) as a pale yellow oil, which was immediately used in the next reaction: ${ }^{1} \mathrm{H}$ NMR $\left(500 \mathrm{MHz}, \mathrm{C}_{6} \mathrm{D}_{6}\right) \delta 6.53(\mathrm{~s}, 1 \mathrm{H}), 5.55$ (ddd, $\left.J=7.5,10.2,17.3 \mathrm{~Hz}, 1 \mathrm{H}\right), 4.92$ (ddd, $J=1.3,1.7,17.3 \mathrm{~Hz}, 1 \mathrm{H}$ ), 4.89 (ddd, $J=0.97,1.7,10.2 \mathrm{~Hz}, 1 \mathrm{H}$ ), 4.63 (s, $1 \mathrm{H}$ ), 4.24 (s, 1 H), $3.91(\mathrm{~m}, 1 \mathrm{H}), 3.45$ (s, $3 \mathrm{H}), 3.21(\mathrm{~s}, 3 \mathrm{H}), 2.16(\mathrm{dq}, \mathrm{J}=2.9,7.0 \mathrm{~Hz}, 1 \mathrm{H}), 2.10(\mathrm{~s}, 3 \mathrm{H}), 1.94$ (apparent septet, $J=7.2 \mathrm{~Hz}, 1 \mathrm{H}), 1.76(\mathrm{~m}, 1 \mathrm{H}), 1.50(\mathrm{~d}, \mathrm{~J}=7.0 \mathrm{~Hz}, 3 \mathrm{H}), 1.45-1.25(\mathrm{~m}, 2 \mathrm{H})$, $1.20(\mathrm{~m}, 1 \mathrm{H}), 1.08(\mathrm{~s}, 9 \mathrm{H}), 0.89(\mathrm{~d}, \mathrm{~J}=6.7 \mathrm{~Hz}, 3 \mathrm{H}), 0.24(\mathrm{~s}, 3 \mathrm{H}), 0.21(\mathrm{~s}, 3 \mathrm{H})$.<smiles>C=C[C@H](C)CC[C@H]1O[C@@H](c2c(Br)c(OC)c(C)c(OC)c2OC(F)(F)C(C)(C)C)CC(=O)[C@@H]1C</smiles>

Pyranone (+)-25: To a solution of $24(1.6 \mathrm{~g}, 2.9 \mathrm{mmol})$ in dichloromethane (36 $\mathrm{mL})$ was added a $1 \mathrm{M}$ solution of $\mathrm{Me}_{2} \mathrm{AlCl}(3.6 \mathrm{~mL}, 3.6 \mathrm{mmol})$ in dichloromethane, at $-78{ }^{\circ} \mathrm{C}$. The reaction mixture was stirred for $10 \mathrm{~min}$, then was quenched at $-78{ }^{\circ} \mathrm{C}$ with $\mathrm{Et}_{3} \mathrm{~N}(1.5 \mathrm{~mL})$, followed by saturated 
aqueous $\mathrm{NaHCO}_{3}(20 \mathrm{~mL})$. The reaction mixture was then diluted with dichloromethane $(100 \mathrm{~mL})$, allowed to warm to $0-5{ }^{\circ} \mathrm{C}$, and neutralized with $1 \mathrm{~N}$ aqueous $\mathrm{HCl}$ to $\mathrm{pH}$ 2. The aqueous phase was extracted with dichloromethane $(3 \times 100 \mathrm{~mL})$, and the combined organic extracts were dried over $\mathrm{Na}_{2} \mathrm{SO}_{4}$, and then concentrated in vacuo. Flash chromatography purification with $5 \%$ ethyl acetate in hexanes as eluent afforded pyranone (+)-25 (1.4 g, 85\% yield) as a colorless oil: $[\alpha]_{\mathrm{D}}^{23}$ +30.8 (c 0.250, $\left.\mathrm{CHCl}_{3}\right)$; IR $\left(\mathrm{CHCl}_{3}\right) 2931$ (s), 1716 (s), 1457 (m), 1387 (m), 1259 (m), 1096 (m), 840 (s) cm ${ }^{-1} ;{ }^{1} \mathrm{H}$ NMR $\left(500 \mathrm{MHz}, \mathrm{C}_{6} \mathrm{D}_{6}\right) \delta 5.56$ (dd, $\mathrm{J}=3.0,12.3 \mathrm{~Hz}, 1 \mathrm{H}$ ), 5.53 (ddd, $\mathrm{J}=7.5,9.8$, $17.2 \mathrm{~Hz}, 1 \mathrm{H}$ ), 4.89 (ddd, $\mathrm{J}=1.1,1.8,17.2 \mathrm{~Hz}, 1 \mathrm{H}$ ), 4.88 (ddd, $J=0.7,1.7,9.8 \mathrm{~Hz}, 1 \mathrm{H}$ ), 3.78 (dd, J = 12.4, 14.4 Hz, 1 H), 3.69 (m, 1 H), 3.43 (s, 3 H), 3.25 (s, 3 H), 2.45 (m, 1 H), 2.35 (ddd, J = 1.2, 3.0, $14.4 \mathrm{~Hz}, 1 \mathrm{H}$ ), 2.10 (s, $3 \mathrm{H}), 1.95$ (apparent septet, J = 7.5 Hz, $1 \mathrm{H}), 1.79(\mathrm{~m}, 1 \mathrm{H}), 1.40$ (m, 1 H), $1.28(m, 1 \mathrm{H}), 1.23(\mathrm{~d}, \mathrm{~J}=7.2 \mathrm{~Hz}, 3 \mathrm{H}), 1.15(\mathrm{~m}, 1 \mathrm{H}), 1.01(\mathrm{~s}, 9 \mathrm{H}), 0.87(\mathrm{~d}, \mathrm{~J}=6.7 \mathrm{~Hz}$, $3 \mathrm{H}), 0.19$ (s, $3 \mathrm{H}), 0.16$ (s, $3 \mathrm{H}) ;{ }^{13} \mathrm{C}$ NMR $\left(125 \mathrm{MHz}, \mathrm{C}_{6} \mathrm{D}_{6}\right) \delta 208.8,151.7,149.7,144.3,144.0$, $128.9,126.6,113.7,112.9,80.6,74.0,59.8,59.6,49.7,42.0,38.0,32.9,30.1,26.2,20.2,18.7$, 10.8, 10.4, -3.8, -4.4; high resolution mass spectrum $(\mathrm{ES}+) \mathrm{m} / \mathrm{z} 577.1989\left[(\mathrm{M}+\mathrm{Na})^{+}\right.$; calcd for $\left.\mathrm{C}_{27} \mathrm{H}_{43} \mathrm{O}_{5} \mathrm{BrSiNa}: 577.1960\right]$.<smiles>C=C[C@H](C)CC[C@H]1O[C@@H](c2c(Br)c(OC)c(C)c(OC)c2OC(F)(F)F)[C@H](C(F)(F)F)C(=O)C1C</smiles>

$\alpha, \alpha^{\prime}$-Dimethylketone $(+)-26$ : A pre-cooled $\left(-78^{\circ} \mathrm{C}\right)$ solution of $(+)-25(2.1 \mathrm{~g}, 3.8 \mathrm{mmol})$ in THF $(20 \mathrm{~mL})$ was added to a $1 \mathrm{M}$ solution of LHMDS $(4.9 \mathrm{~mL}, 4.9 \mathrm{mmol})$ in hexanes at $-78{ }^{\circ} \mathrm{C}$. The reaction mixture was stirred for $30 \mathrm{~min}$ and then for an additional $1 \mathrm{~h}$ at $-10^{\circ} \mathrm{C}$. A pre-cooled $(-10$ $\left.{ }^{\circ} \mathrm{C}\right)$ solution of Mel $(0.7 \mathrm{~mL}, 11.3 \mathrm{mmol})$ in THF $(11 \mathrm{~mL})$ was next added, and the reaction mixture was stirred for $3 \mathrm{~h}$ at $-20-0{ }^{\circ} \mathrm{C}$. The reaction was quenched with saturated aqueous $\mathrm{NH}_{4} \mathrm{Cl}(2$ $\mathrm{mL})$, allowed to reach room temperature, and extracted with $\mathrm{Et}_{2} \mathrm{O}(3 \times 100 \mathrm{~mL})$. The combined organic extracts were dried over $\mathrm{Na}_{2} \mathrm{SO}_{4}$, and then concentrated in vacuo. Flash chromatography 
purification with 4\% ethyl acetate in hexanes as eluent afforded (+)-26 (1.5 g, 70\% yield) as a colorless oil: $[\alpha]_{\mathrm{D}}^{23}+41.0$ (c 1.585, $\left.\mathrm{CHCl}_{3}\right)$; IR $\left(\mathrm{CHCl}_{3}\right) 2955$ (s), 2932 (s), 2858 (m), 1712 (s), 1448 (s), 1387 (m), $1238(\mathrm{~m}), 1086(\mathrm{~m}), 840$ (s) cm ${ }^{-1} ;{ }^{1} \mathrm{H}$ NMR (500 MHz, $\left.\mathrm{C}_{6} \mathrm{D}_{6}\right) \delta 5.55$ (ddd, $\mathrm{J}=$ 7.5, 10.3, $17.4 \mathrm{~Hz}, 1 \mathrm{H}), 5.15$ (d, J = $11.1 \mathrm{~Hz}, 1 \mathrm{H}), 4.91$ (ddd, J = 1.1, 1.7, $17.1 \mathrm{~Hz}, 1 \mathrm{H}$ ), 4.89 (ddd, $J=0.8,1.7,10.3 \mathrm{~Hz}, 1 \mathrm{H}$ ), 3.82 (dq, J = 6.6, $11.0 \mathrm{~Hz}, 1 \mathrm{H}), 3.71(\mathrm{~m}, 1 \mathrm{H}), 3.41(\mathrm{~s}, 3 \mathrm{H}), 3.28$ (s, 3 H), 2.53 (dq, J = 2.5, 7.2 Hz, 1 H), 2.30 (s, 3 H), 1.98 (apparent septet, $J=7.3 \mathrm{~Hz}, 1 \mathrm{H}$ ), 1.80 (m, $1 \mathrm{H}), 1.45(\mathrm{~m}, 1 \mathrm{H}), 1.29(\mathrm{~m}, 1 \mathrm{H}), 1.28(\mathrm{~d}, \mathrm{~J}=7.2 \mathrm{~Hz}, 3 \mathrm{H}), 1.10(\mathrm{~m}, 1 \mathrm{H}), 1.01(\mathrm{~d}, J=6.6 \mathrm{~Hz}$, $3 \mathrm{H}), 1.00(\mathrm{~s}, 9 \mathrm{H}), 0.91(\mathrm{~d}, \mathrm{~J}=6.6 \mathrm{~Hz}, 3 \mathrm{H}), 0.23(\mathrm{~s}, 3 \mathrm{H}), 0.11(\mathrm{~s}, 3 \mathrm{H}) ;{ }^{13} \mathrm{C}$ NMR $(125 \mathrm{MHz}$, $\left.\mathrm{C}_{6} \mathrm{D}_{6}\right) \delta 211.5,151.8,149.8,144.7,144.3,129.0,126.6,113.2,112.9,80.7,79.4,59.8,59.5$, $50.0,43.5,38.0,33.0,30.1,26.2,20.2,18.6,11.1,10.4,9.4,-3.7,-4.7$; high resolution mass spectrum (ES+) $\mathrm{m} / \mathrm{z} 591.2108$ [(M+Na) ${ }^{+}$; calcd for $\left.\mathrm{C}_{28} \mathrm{H}_{45} \mathrm{O}_{5} \mathrm{BrSiNa}: 591.2117\right]$.

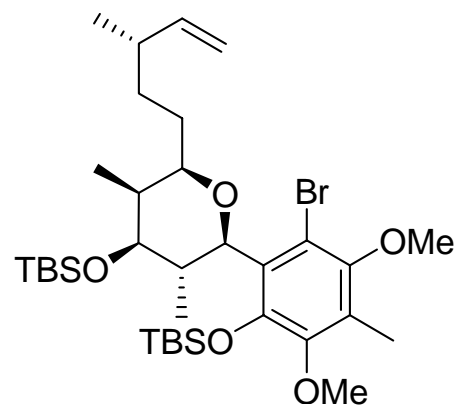

Protected alcohol (+)-4: To a solution of $(+)-26(1.5 \mathrm{~g}, 2.6 \mathrm{mmol})$ in ethanol $(26 \mathrm{~mL})$ at $-78{ }^{\circ} \mathrm{C}$ was slowly added $\mathrm{NaBH}_{4}(0.2 \mathrm{~g}, 3.3 \mathrm{mmol})$, and then the reaction mixture was stirred for $2.5 \mathrm{~h}$ at the same temperature. The reaction mixture was quenched with saturated aqueous $\mathrm{NH}_{4} \mathrm{Cl}(15$ $\mathrm{mL}$ ), allowed to reach room temperature, and extracted with chloroform $(3 \times 100 \mathrm{~mL})$. The combined organic extracts were dried over $\mathrm{Na}_{2} \mathrm{SO}_{4}$, and then concentrated in vacuo to give a crude mixture of the corresponding alcohols (5:1 dr). Flash chromatography separation with ethyl acetate-hexanes $(1: 49 \rightarrow 1: 9)$ as eluent afforded the pure $\mathrm{C}(7)$ alcohol $(1.2 \mathrm{~g}, 81 \%$ yield $)$ as a colorless oil: $[\alpha]_{\mathrm{D}}^{23}+28.1$ (c 2.140, $\mathrm{CHCl}_{3}$ ); IR $\left(\mathrm{CHCl}_{3}\right) 3329$ (br), 2956 (s), 2930 (s), 2857 (m), 1446 (s), 1405 (s), 1384 (m), 1237 (m), 1106 (s), 1024 (m), 839 (s) cm ${ }^{-1} ;{ }^{1} \mathrm{H}$ NMR (500 MHz, $\left.\mathrm{C}_{6} \mathrm{D}_{6}\right) \delta 5.63$ (ddd, $\mathrm{J}=7.5,10.3,17.4 \mathrm{~Hz}, 1 \mathrm{H}$ ), 4.95 (apparent td, $\mathrm{J}=1.5,17.3 \mathrm{~Hz}, 1 \mathrm{H}$ ), 4.92 
(apparent td, J = 0.8, 10.3 Hz, $1 \mathrm{H}), 4.85(\mathrm{~d}, \mathrm{~J}=10.6 \mathrm{~Hz}, 1 \mathrm{H}), 3.48(\mathrm{~m}, 1 \mathrm{H}), 3.41(\mathrm{~s}, 3 \mathrm{H}), 3.39$ (m, $1 \mathrm{H}), 3.33$ (s, $3 \mathrm{H}), 2.70$ (m, $1 \mathrm{H}), 2.15$ (s, $3 \mathrm{H}$ ), 2.06 (apparent septet, J = 7.3 Hz, $1 \mathrm{H}$ ), 1.85 (m, $1 \mathrm{H}), 1.77(\mathrm{~m}, 1 \mathrm{H}), 1.52(\mathrm{~m}, 1 \mathrm{H}), 1.43(\mathrm{~m}, 1 \mathrm{H}), 1.25$ (d, J = 6.9 Hz, $3 \mathrm{H}), 1.23(\mathrm{~m}, 1 \mathrm{H}), 1.08$ (s, 9 H), 0.96 (dd, J = 5.8, 10.1 Hz, 1 H), $0.95(d, J=6.7 \mathrm{~Hz}, 3 \mathrm{H}), 0.85$ (d, J = $6.5 \mathrm{~Hz}, 3 \mathrm{H}), 0.28$ (s, $3 \mathrm{H}), 0.17$ (s, $3 \mathrm{H}) ;{ }^{13} \mathrm{C}$ NMR $\left(125 \mathrm{MHz}, \mathrm{C}_{6} \mathrm{D}_{6}\right) \delta 151.7,149.6,144.7,144.5,129.7,125.9$, $113.6,112.7,80.2,78.4,78.0,59.7,59.5,39.7,38.1,35.1,33.4,31.1,26.3,20.2,18.7,13.2$, 10.4, 7.1, -3.6, -4.7; high resolution mass spectrum (ES+) $\mathrm{m} / \mathrm{z} 593.2295\left[(\mathrm{M}+\mathrm{Na})^{+}\right.$; calcd for $\mathrm{C}_{28} \mathrm{H}_{47} \mathrm{O}_{5} \mathrm{BrSiNa}:$ 593.2273].

To a solution of the above alcohol $(1.2 \mathrm{~g}, 2.1 \mathrm{mmol})$ in dichloromethane $(7 \mathrm{~mL})$ at $-10{ }^{\circ} \mathrm{C}$ was added dropwise a mixture of TBSOTf $(0.73 \mathrm{~mL}, 3.2 \mathrm{mmol})$ and 2,6-lutidine $(0.5 \mathrm{~mL}, 4.2 \mathrm{mmol})$ in dichloromethane $(14 \mathrm{~mL})$. The reaction mixture was stirred for $1 \mathrm{~h}$ at $-10^{\circ} \mathrm{C}$, and then quenched with saturated aqueous $\mathrm{NaHCO}_{3}(29 \mathrm{~mL})$, allowed to reach room temperature, and extracted with $\mathrm{Et}_{2} \mathrm{O}(3 \times 100 \mathrm{~mL})$. The combined organic extracts were dried over $\mathrm{Na}_{2} \mathrm{SO}_{4}$, and then concentrated in vacuo. Flash chromatography purification with $3 \%$ ethyl acetate in hexanes as eluent afforded (+)-4 (1.38 g, 95\% yield) as a colorless oil: $[\alpha]_{\mathrm{D}}^{23}+35.4\left(\mathrm{c} 1.090, \mathrm{CHCl}_{3}\right)$; IR $\left(\mathrm{CHCl}_{3}\right) 2956$ (s), 2930 (s), $2857(\mathrm{~m}), 1447$ (m), 1404 (m), 1387 (m), 1251 (m), 1107 (s), 1075 (s), $1026(\mathrm{~m}), 836$ (s) cm ${ }^{-1} ;{ }^{1} \mathrm{H}$ NMR $\left(500 \mathrm{MHz}, \mathrm{C}_{6} \mathrm{D}_{6}\right) \delta 5.64$ (ddd, $\mathrm{J}=7.5,10.3,17.5 \mathrm{~Hz}, 1 \mathrm{H}$ ), 4.97 (apparent td, $J=1.4,17.5 \mathrm{~Hz}, 1 \mathrm{H}$ ), 4.95 (d, J = 10.6 Hz, $1 \mathrm{H}$ ), 4.92 (ddd, J = 0.7, 1.1, 10.3 Hz, 1 H), 3.70 (dd, J = 4.6, 9.9 Hz, 1 H), 3.64 (ddd, J = 2.0, 4.3, 8.5 Hz, 1 H), 3.41 (s, 3 H), 3.35 (s, 3 H), 2.95 (qdd, J = 6.6, 9.9, $10.6 \mathrm{~Hz}, 1 \mathrm{H}$ ), 2.15 (s, $3 \mathrm{H}$ ), 2.09 (apparent septet, $J=6.9 \mathrm{~Hz}, 1 \mathrm{H}$ ), 1.98 (ddq, $J$ = 2.0, 4.6, 6.7 Hz, 1 H), $1.91(m, 1 \mathrm{H}), 1.65(\mathrm{~m}, 1 \mathrm{H}), 1.46(\mathrm{~m}, 1 \mathrm{H}), 1.45(\mathrm{~d}, \mathrm{~J}=6.9$ Hz, 3 H), 1.32 (m, 1 H), 1.10 (s, 9 H), 1.02 (s, 9 H), 0.97 (d, J = 6.7 Hz, 3 H), 0.91 (d, J = 6.6 Hz, $3 \mathrm{H}), 0.30$ (s, $3 \mathrm{H}), 0.19$ (s, $3 \mathrm{H}), 0.15$ (s, $6 \mathrm{H}) ;{ }^{13} \mathrm{C}$ NMR $\left(125 \mathrm{MHz}, \mathrm{C}_{6} \mathrm{D}_{6}\right) \delta$ 151.8, 149.6, 144.7, $144.4,129.8,125.9,113.6,112.7,80.3,79.4,78.4,59.7,59.4,41.0,38.1,35.3,33.6,31.3,26.3$, 26.0, 20.2, 18.7, 18.4, 13.7, 10.4, 7.5, -3.6, -4.0, -4.6, -4.7; high resolution mass spectrum (ES+) $\mathrm{m} / \mathrm{z} 707.3110$ [(M+Na) ${ }^{+}$; calcd for $\left.\mathrm{C}_{34} \mathrm{H}_{61} \mathrm{O}_{5} \mathrm{BrSiNa}: 707.3138\right]$. 


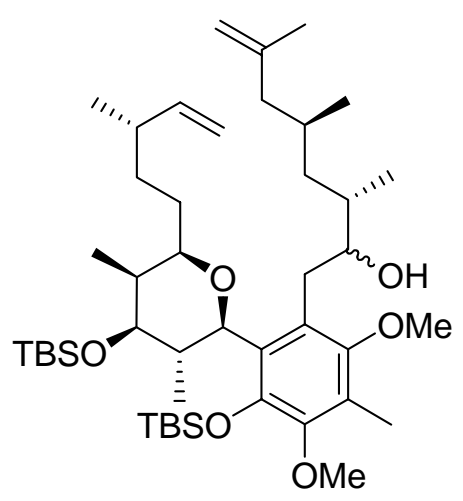

Alcohols 27: To a solution of $(+)-4(77 \mathrm{mg}, 0.11 \mathrm{mmol})$ in THF $(0.6 \mathrm{~mL})$ was added $t$-BuLi $(1.7 \mathrm{M}$ in pentane, $0.13 \mathrm{~mL}, 0.23 \mathrm{mmol}$ ) at $-78{ }^{\circ} \mathrm{C}$, and then the reaction mixture was stirred for $5 \mathrm{~min}$ at the same temperature. A solution of 5 (38 $\mathrm{mg}, 0.23 \mathrm{mmol})$ in THF $(0.2 \mathrm{~mL})$ was then added, followed by $\mathrm{BF}_{3} \cdot \mathrm{OEt}_{2}(17 \mu \mathrm{L}, 0.14 \mathrm{mmol})$, and the reaction mixture was stirred at $-78{ }^{\circ} \mathrm{C}$ for an additional $3 \mathrm{~h}$. The reaction mixture was quenched with $\mathrm{MeOH}(0.4 \mathrm{~mL})$, then saturated aqueous $\mathrm{NH}_{4} \mathrm{Cl}(0.4 \mathrm{~mL})$, allowed to reach room temperature, and extracted with $\mathrm{Et}_{2} \mathrm{O}(3 \times 50 \mathrm{~mL})$. The combined organic extracts were dried over $\mathrm{Na}_{2} \mathrm{SO}_{4}$, and then concentrated in vacuo. Preparative thin-layer chromatography purification with $5 \%$ ethyl acetate in hexanes as eluent afforded the alcohols 27 (53 mg, 60\% yield, 2:1 dr) as a colorless oil: IR ( $\left.\mathrm{CHCl}_{3}\right) 3481$ (br), 2956 (s), 2930 (s), 2857 (s), 1454 (s), 1410 (m), 1386 (s), 1333 (m), 1253 (s), 1110 (s), 1071 (s), 1005 (m), 885 (m), 837 (s), $778(\mathrm{~m}) \mathrm{cm}^{-1} ;{ }^{1} \mathrm{H}$ NMR (The asterisk denotes the minor diastereomer, $\left.500 \mathrm{MHz}, \mathrm{C}_{6} \mathrm{D}_{6}\right) \delta$ 5.83* (ddd, $J=7.7,10.4,17.6 \mathrm{~Hz}, 1 \mathrm{H}$ ), 5.71 (ddd, $J=7.6,10.4,17.8 \mathrm{~Hz}, 1 \mathrm{H}$ ), 5.06* (apparent $\mathrm{td}, J=1.2,17.6 \mathrm{~Hz}, 1 \mathrm{H}$ ), 5.02 (apparent td, $J=1.3,17.3 \mathrm{~Hz}, 1 \mathrm{H}), 4.99^{\star}(\mathrm{br} \mathrm{d}, J=10.3 \mathrm{~Hz}, 1 \mathrm{H}$ ), 4.97* (d, J = 9.5 Hz, $1 \mathrm{H}), 4.96(\mathrm{br} \mathrm{d}, J=10.4 \mathrm{~Hz}, 1 \mathrm{H}), 4.92(\mathrm{~d}, \mathrm{~J}=10.8 \mathrm{~Hz}, 1 \mathrm{H}), 4.82^{\star}$ (br s, 1 H), 4.81 (apparent s, 2 H), 4.81* (overlapped, 1 H), 4.19 (d, J = 5.6 Hz, 1 H), 4.07 (m, 1 H), 4.07* (overlapped, $1 \mathrm{H}), 3.93^{*}(\mathrm{~m}, 1 \mathrm{H}), 3.66(\mathrm{dd}, \mathrm{J}=4.9,10.0 \mathrm{~Hz}, 1 \mathrm{H}), 3.63^{\star}(\mathrm{dd}, \mathrm{J}=5.1,10.3 \mathrm{~Hz}, 1$ H), 3.60* (apparent td, J = 2.5, 9.2 Hz, $1 \mathrm{H}$ ), 3.53 (ddd, $J=1.8,4.0,8.9 \mathrm{~Hz}, 1 \mathrm{H}), 3.44^{\star}(\mathrm{s}, 3 \mathrm{H}$ ), $3.40(\mathrm{~s}, 3 \mathrm{H}), 3.38^{*}(\mathrm{~s}, 3 \mathrm{H}), 3.27(\mathrm{~m}, 1 \mathrm{H}), 3.25(\mathrm{~s}, 3 \mathrm{H}), 3.20^{*}(\mathrm{~m}, 1 \mathrm{H}), 3.17(\mathrm{dd}, \mathrm{J}=2.3,13.4$ $\mathrm{Hz}, 1 \mathrm{H}), 3.06^{\star}(\mathrm{dd}, \mathrm{J}=10.5,13.3 \mathrm{~Hz}, 1 \mathrm{H}), 2.96$ (dd, $\left.J=10.1,13.5 \mathrm{~Hz}, 1 \mathrm{H}\right), 2.93^{\star}(\mathrm{br} d, J=13.4$ $\mathrm{Hz}, 1 \mathrm{H}), 2.26$ (m, $1 \mathrm{H}), 2.26^{\star}$ (overlapped, $\left.1 \mathrm{H}\right), 2.16^{\star}(\mathrm{s}, 3 \mathrm{H}), 2.13(\mathrm{~m}, 1 \mathrm{H}), 2.13^{\star}$ (overlapped, $1 \mathrm{H}), 2.07$ (s, $3 \mathrm{H}), 2.03(\mathrm{~m}, 1 \mathrm{H}), 2.01-1.72(\mathrm{~m}, 3 \mathrm{H}), 1.71-1.47^{\star}(\mathrm{m}, 4 \mathrm{H}), 1.62^{\star}(\mathrm{s}, 3 \mathrm{H}), 1.60(\mathrm{~s}$, $3 \mathrm{H}), 1.43-1.19(\mathrm{~m}, 2 \mathrm{H}), 1.24^{*}(\mathrm{~d}, \mathrm{~J}=7.0 \mathrm{~Hz}, 3 \mathrm{H}), 1.21(\mathrm{~d}, \mathrm{~J}=7.0 \mathrm{~Hz}, 3 \mathrm{H}), 1.14(\mathrm{~m}, 1 \mathrm{H}), 1.12$ 
$(\mathrm{d}, J=6.7 \mathrm{~Hz}, 3 \mathrm{H}), 1.09^{*}(\mathrm{~d}, \mathrm{~J}=6.8 \mathrm{~Hz}, 3 \mathrm{H}), 1.03(\mathrm{~s}, 9 \mathrm{H}), 1.01^{*}(\mathrm{~s}, 9 \mathrm{H}), 0.96(\mathrm{~d}, \mathrm{~J}=6.4 \mathrm{~Hz}, 3$ H), $0.94(d, J=7.0 \mathrm{~Hz}, 3 \mathrm{H}), 0.93(\mathrm{~s}, 9 \mathrm{H}), 0.92^{\star}(\mathrm{s}, 9 \mathrm{H}), 0.91^{\star}(\mathrm{d}, J=6.7 \mathrm{~Hz}, 3 \mathrm{H}), 0.82(\mathrm{~d}, J=$ $6.7 \mathrm{~Hz}, 3 \mathrm{H}), 0.80^{\star}(\mathrm{d}, \mathrm{J}=6.6 \mathrm{~Hz}, 3 \mathrm{H}), 0.20(\mathrm{~s}, 3 \mathrm{H}), 0.20$ (s, $\left.3 \mathrm{H}\right), 0.17^{\star}(\mathrm{s}, 3 \mathrm{H}), 0.05(\mathrm{~s}, 3 \mathrm{H})$, 0.04 (s, $6 \mathrm{H}), 0.04^{\star}$ (overlapped s, $\left.3 \mathrm{H}\right), 0.03^{\star}(\mathrm{s}, 3 \mathrm{H}), 0.02^{\star}(\mathrm{s}, 3 \mathrm{H}) ;{ }^{13} \mathrm{C}$ NMR $\left(125 \mathrm{MHz}, \mathrm{C}_{6} \mathrm{D}_{6}\right) \delta$ $153.7^{\star}, 152.9,148.8^{\star}, 148.6,144.9^{\star}, 144.8,144.7,144.6^{\star}, 143.8,143.0^{\star}, 129.7,129.3^{\star}, 129.2^{\star}$ $129.1,125.1^{\star}, 124.2,113.0^{\star}, 112.9,111.9,81.3^{\star}, 80.7,79.3,78.9,78.6^{\star}, 77.6,76.7^{\star}, 59.7^{\star}, 59.6$, $59.4,59.3^{\star}, 47.7^{\star}, 47.6,42.1,40.9^{\star}, 40.8,39.7^{\star}, 39.0{ }^{*}, 38.5^{\star}, 38.4^{\star}, 38.3,38.2,37.4,34.0,33.8$, $33.6^{\star}, 32.1^{\star}, 31.3,31.1^{\star}, 28.4^{\star}, 28.3,26.4,26.1,26.0^{\star}, 22.4,22.3^{\star}, 20.4^{\star}, 20.3,19.5^{\star}, 19.1,18.8$ $18.7^{\star}, 18.4,18.3^{\star}, 15.5^{\star}, 13.7,13.5^{\star}, 12.6,10.2^{\star}, 9.9,7.6^{\star}, 7.5,-3.6,-3.8^{\star},-4.0,-4.6,-4.7^{\star},-4.8,-$ 4.9*; high resolution mass spectrum (ES+) $\mathrm{m} / \mathrm{z} 797.5520\left[(\mathrm{M}+\mathrm{Na})^{+}\right.$; calcd for $\mathrm{C}_{45} \mathrm{H}_{82} \mathrm{O}_{6} \mathrm{Si}_{2} \mathrm{Na}$ : 797.5547].

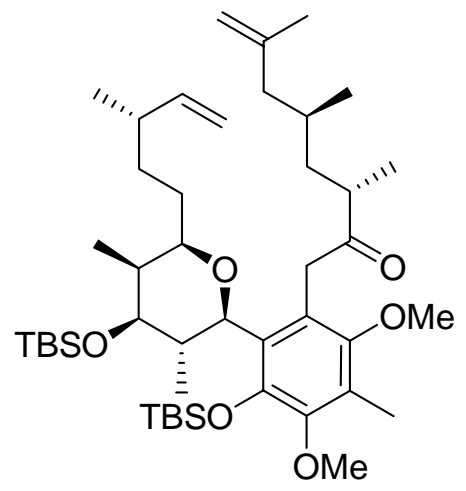

Ketone (+)-29: A solution of $27(14.6 \mathrm{mg}, 0.019 \mathrm{mmol})$, and pyridine $(3 \mu \mathrm{L}, 0.038 \mathrm{mmol})$ in dichloromethane $(0.75 \mathrm{~mL})$ was treated with Dess-Martin periodinane $(32.2 \mathrm{mg}, 0.076 \mathrm{mmol})$ at 0 ${ }^{\circ} \mathrm{C}$, and then the reaction mixture was stirred for $2 \mathrm{~h}$ at room temperature. The reaction mixture was quenched with a 1:1 (v/v) mixture of saturated aqueous $\mathrm{Na}_{2} \mathrm{~S}_{2} \mathrm{O}_{3}$ and saturated aqueous $\mathrm{NaHCO}_{3}(1 \mathrm{~mL})$ and extracted with $\mathrm{Et}_{2} \mathrm{O}(3 \times 5 \mathrm{~mL})$. The combined organic extracts were washed with water and brine (5 mL each), dried over $\mathrm{Na}_{2} \mathrm{SO}_{4}$, and then concentrated in vacuo. Preparative thin-layer chromatography purification with $5 \%$ ethyl acetate in hexanes as eluent afforded (+)-29 (12.8 mg, 88\% yield) as a colorless oil: $[\alpha]_{\mathrm{D}}^{23}+23.2\left(\mathrm{c} 0.190, \mathrm{CHCl}_{3}\right) ; \mathrm{IR}\left(\mathrm{CHCl}_{3}\right)$ $2928(\mathrm{~s}), 2856(\mathrm{~m}), 1709(\mathrm{~m}), 1461(\mathrm{~m}), 1410(\mathrm{~m}), 1384(\mathrm{~m}), 1253(\mathrm{~m}), 1069(\mathrm{~s}), 1005(\mathrm{~m}), 889$ (m), 836 (s), $775(\mathrm{~m}) \mathrm{cm}^{-1} ;{ }^{1} \mathrm{H}$ NMR (500 MHz, $\left.\mathrm{C}_{6} \mathrm{D}_{6}\right) \delta 5.69$ (ddd, $\mathrm{J}=7.5,10.3,17.5 \mathrm{~Hz}, 1 \mathrm{H}$ ), 
5.00 (apparent td, $J=1.4,17.5 \mathrm{~Hz}, 1 \mathrm{H}$ ), 4.96 (d, $\mathrm{J}=10.8 \mathrm{~Hz}, 1 \mathrm{H}$ ), 4.95 (apparent td, $\mathrm{J}=1.1$, $10.3 \mathrm{~Hz}, 1 \mathrm{H}), 4.81$ (br s, $1 \mathrm{H}$ ), 4.76 (br s, $1 \mathrm{H}), 4.41(\mathrm{~d}, \mathrm{~J}=17.2 \mathrm{~Hz}, 1 \mathrm{H}), 4.28(\mathrm{~d}, \mathrm{~J}=17.2 \mathrm{~Hz}, 1$ H), $3.70(\mathrm{dd}, \mathrm{J}=4.7,9.9 \mathrm{~Hz}, 1 \mathrm{H}), 3.60(\mathrm{~m}, 1 \mathrm{H}), 3.43(\mathrm{~s}, 3 \mathrm{H}), 3.34(\mathrm{~s}, 3 \mathrm{H}), 2.82(\mathrm{~m}, 1 \mathrm{H}), 2.19$ (s, 3 H), 2.11 (apparent septet, J = 6.7 Hz, 1 H), 2.09 (m, 1 H), $2.04(m, 1$ H), $1.95(m, 1 \mathrm{H}), 1.82-$ $1.65(\mathrm{~m}, 5 \mathrm{H}), 1.62(\mathrm{~s}, 3 \mathrm{H}), 1.50-1.25(\mathrm{~m}, 6 \mathrm{H}), 1.26(\mathrm{~d}, \mathrm{~J}=6.9 \mathrm{~Hz}, 3 \mathrm{H}), 1.20(\mathrm{~d}, \mathrm{~J}=6.8 \mathrm{~Hz}, 3$ H), $1.12(\mathrm{~s}, 9 \mathrm{H}), 1.00(\mathrm{~d}, J=6.7 \mathrm{~Hz}, 3 \mathrm{H}), 0.99(\mathrm{~s}, 9 \mathrm{H}), 0.82(\mathrm{~d}, J=6.2 \mathrm{~Hz}, 3 \mathrm{H}), 0.32(\mathrm{~s}, 3 \mathrm{H})$, 0.19 (s, $3 \mathrm{H}), 0.13$ (s, $3 \mathrm{H}), 0.12(\mathrm{~s}, 3 \mathrm{H}) ;{ }^{13} \mathrm{C}$ NMR $\left(125 \mathrm{MHz}, \mathrm{C}_{6} \mathrm{D}_{6}\right) \delta 203.5,153.8,149.9,145.3$, $145.1,143.9,129.5,125.6,124.8,113.2,112.4,80.8,79.2,78.8,59.6,59.3,46.7,42.5,41.5$, $40.7,40.5,38.3,38.1,33.5,31.3,29.5,28.2,26.3,25.9,20.0,19.0,18.0,17.6,16.2,13.4,9.9$, 7.2, -3.8, -5.4, -5.0 (2 overlapped signals); high resolution mass spectrum (ES+) $\mathrm{m} / \mathrm{z} 795.5405$ $\left[(\mathrm{M}+\mathrm{Na})^{+}\right.$; calcd for $\left.\mathrm{C}_{45} \mathrm{H}_{80} \mathrm{O}_{6} \mathrm{Si}_{2} \mathrm{Na}: 795.5391\right]$.

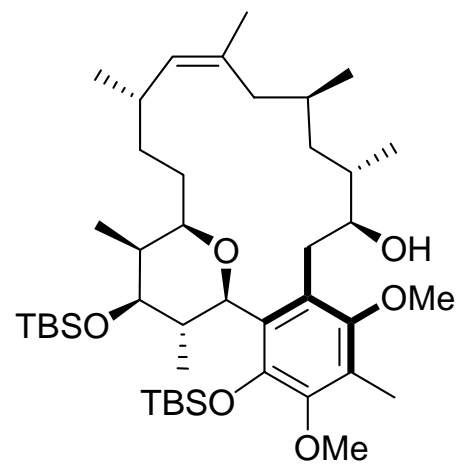

Z Olefin (+)-30: A solution of $27(0.6 \mathrm{~g}, 0.775 \mathrm{mmol})$ and second generation Grubbs' catalyst (66 $\mathrm{mg}, 0.08 \mathrm{mmol}$ ) in dichloromethane was stirred at $50-55^{\circ} \mathrm{C}$ (external temperature) for $2 \mathrm{~d}$. After cooling to room temperature, the reaction mixture was concentrated in vacuo and purified by flash chromatography with $3 \%$ ethyl acetate in hexanes as eluent to afford $(+)-30(0.33 \mathrm{~g}, 57 \%$ yield $)$ as a white solid: m.p. $187-188{ }^{\circ} \mathrm{C} ;[\alpha]_{\mathrm{D}}^{23}+44.9\left(\mathrm{c} \mathrm{0.550,} \mathrm{CHCl}_{3}\right)$; IR $\left(\mathrm{CHCl}_{3}\right) 3508$ (br), 2955 (s), $2929(\mathrm{~s}), 2856(\mathrm{~m}), 1461(\mathrm{~s}), 1410(\mathrm{~m}), 1387(\mathrm{~m}), 1252(\mathrm{~s}), 1074(\mathrm{~s}), 1004(\mathrm{~m}), 886(\mathrm{~m}), 836(\mathrm{~s})$, $774(\mathrm{~m}) \mathrm{cm}^{-1} ;{ }^{1} \mathrm{H}$ NMR $\left(500 \mathrm{MHz}, \mathrm{C}_{6} \mathrm{D}_{6}\right) \delta 5.22(\mathrm{~d}, \mathrm{~J}=9.7 \mathrm{~Hz}, 1 \mathrm{H}), 4.91(\mathrm{~d}, \mathrm{~J}=10.5 \mathrm{~Hz}, 1 \mathrm{H})$, 4.24 (s, $1 \mathrm{H}$ ), 4.06 (d, J = 8.6 Hz, $1 \mathrm{H}$ ), 3.67 (dd, J = 4.8, $9.9 \mathrm{~Hz}, 1 \mathrm{H}$ ), 3.57 (apparent td, $\mathrm{J}=2.2$, $10.5 \mathrm{~Hz}, 1 \mathrm{H}), 3.52(\mathrm{~d}, \mathrm{~J}=13.1 \mathrm{~Hz}, 1 \mathrm{H}), 3.44(\mathrm{~s}, 3 \mathrm{H}), 3.28(\mathrm{~s}, 3 \mathrm{H}), 2.83(\mathrm{dd}, \mathrm{J}=8.8,13.2 \mathrm{~Hz}, 1$ H), $2.60(\mathrm{~d}, \mathrm{~J}=11.2 \mathrm{~Hz}, 1 \mathrm{H}), 2.33(\mathrm{~m}, 1 \mathrm{H}), 2.10(\mathrm{~s}, 3 \mathrm{H}), 2.06(\mathrm{~m}, 1 \mathrm{H}), 1.99-1.90(\mathrm{~m}, 4 \mathrm{H}), 1.82$ 
(m, 1 H), 1.72 (br s, 3 H), $1.81(m, 1$ H), 1.58-1.52 (m, 2 H), $1.34(m, 1 \mathrm{H}), 1.32$ (d, J = 6.0 Hz, 3 H), $1.30(\mathrm{~d}, \mathrm{~J}=6.7 \mathrm{~Hz}, 3 \mathrm{H}), 1.21(\mathrm{~m}, 1 \mathrm{H}), 1.15(\mathrm{~d}, \mathrm{~J}=6.5 \mathrm{~Hz}, 3 \mathrm{H}), 1.08(\mathrm{~s}, 9 \mathrm{H}), 1.07$ (d, J = 6.5 Hz, 3 H), $1.02(\mathrm{~s}, 9 \mathrm{H}), 0.82(\mathrm{~d}, \mathrm{~J}=6.6 \mathrm{~Hz}, 3 \mathrm{H}), 0.20(\mathrm{~s}, 3 \mathrm{H}), 0.14(\mathrm{~s}, 3 \mathrm{H}), 0.13(\mathrm{~s}, 3 \mathrm{H}), 0.10$ (s, $3 \mathrm{H}) ;{ }^{13} \mathrm{C}$ NMR $\left(125 \mathrm{MHz}, \mathrm{C}_{6} \mathrm{D}_{6}\right) \delta 152.4,148.6,143.6,135.9,133.0,130.6,129.4,124.3,80.1$, $79.0,78.1,74.7,60.1,59.4,43.6,42.8,41.3,39.7,39.3,35.0,34.9,34.5,34.4,33.6,26.4,26.3$, 26.0, 23.2, 20.6, 18.7, 18.4, 14.4, 13.4, 9.8, 7.4, -3.8, -3.9, -4.7, -4.9; high resolution mass spectrum (ES+) $\mathrm{m} / \mathrm{z} 769.5228\left[(\mathrm{M}+\mathrm{Na})^{+}\right.$; calcd for $\left.\mathrm{C}_{43} \mathrm{H}_{78} \mathrm{O}_{6} \mathrm{Si}_{2} \mathrm{Na}: 769.5234\right]$.

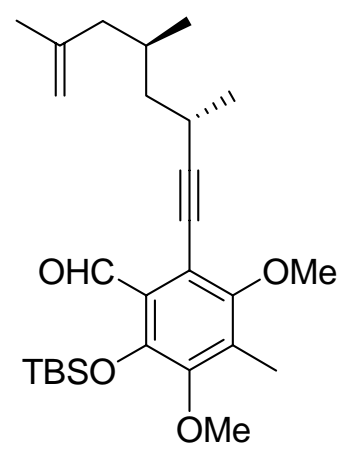

Alkyne (+)-31: To an argon purged (3 times) flask containing $\mathrm{PdCl}_{2}\left(\mathrm{PPh}_{3}\right)_{2}$ (4.5 mg, $\left.5 \mathrm{~mol} \%\right)$, Cul (0.73 mg, $3 \mathrm{~mol} \%)$, and aldehyde 6 (50 mg, $0.128 \mathrm{mmol}$ ) was added degassed diisopropylamine (freeze-thawed 3 times) via cannula. Degassed alkyne (+)-10 (37 $\mu \mathrm{L}, 0.192 \mathrm{mmol})$ was added quickly, and the reaction mixture was heated at $60{ }^{\circ} \mathrm{C}$ for $6.5 \mathrm{~h}$. The solvent was removed in vacuo and the product was purified by flash chromatography (hexanes/EtOAc 97:3) to provide (+)-31 (50 mg, 85\% yield) as a yellow oil: $[\alpha]_{D}^{23}+23.6\left(c\right.$ 1.500, $\left.\mathrm{C}_{6} \mathrm{H}_{6}\right)$; IR (film) 2965 (s), 2930 (s), 2858 (m), 1698 (s), 1647 (w), 1567 (w), 1458 (s), 1393 (s), 1284 (s), 1254 (s), 1101 (s); ${ }^{1} \mathrm{H}$ NMR (500 MHz, $\left.\mathrm{CDCl}_{3}\right) \delta 10.45$ (s, $\left.1 \mathrm{H}\right), 4.84(\mathrm{br} \mathrm{s}, 1 \mathrm{H}), 4.80$ (br s, $\left.1 \mathrm{H}\right), 3.85$ (s, $\left.3 \mathrm{H}\right), 3.69(\mathrm{~s}, 3 \mathrm{H})$, 2.85 (m, $1 \mathrm{H}), 2.22(\mathrm{~s}, 3 \mathrm{H}), 2.14(\mathrm{dd}, \mathrm{J}=5.5,13.3 \mathrm{~Hz}, 1 \mathrm{H}), 1.92(\mathrm{~m}, 1 \mathrm{H}), 1.79$ (dd, $J=8.8,13.3$ Hz, 1 H), 1.70 (s, 3 H), 1.54-1.44 (m, 2 H), 1.29 (d, J = 6.9 Hz, 3 H), 1.00 (s, 9 H), 0.90 (d, J = 6.5 $\mathrm{Hz}, 3 \mathrm{H}), 0.16(\mathrm{~s}, 6 \mathrm{H}) ;{ }^{13} \mathrm{C}$ NMR $\left(125 \mathrm{MHz}, \mathrm{CDCl}_{3}\right) \delta 185.2,155.1,150.4,146.5,144.5,132.5$, $127.1,114.9,111.6,104.9,73.6,60.4,60.1,45.4,44.3,28.6,25.8,24.8,22.2,20.7,19.7,18.6$, 10.2, -4.4 (two overlapped signals); high resolution mass spectrum (ES+) $\mathrm{m} / \mathrm{z} 481.2735$ $\left[(\mathrm{M}+\mathrm{Na})^{+}\right.$; calcd for $\left.\mathrm{C}_{27} \mathrm{H}_{42} \mathrm{NaO}_{4} \mathrm{Si}: 481.2750\right]$. 


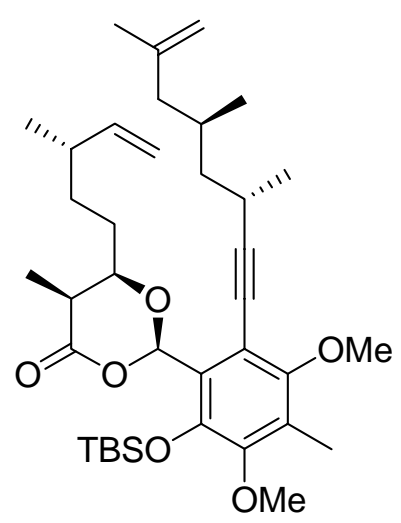

Dioxanone (+)-32: To a solution of hydroxyacid (+)-7 (20.5 mg, $0.11 \mathrm{mmol}),(+)-31$ (39 mg, 0.085 $\mathrm{mmol})$, and isopropoxytrimethylsilane $(60 \mu \mathrm{L}, 0.34 \mathrm{mmol})$ was added TMSOTf $(0.15 \mu \mathrm{L}, 0.85$ $\mu \mathrm{mol}$ ) at $-78{ }^{\circ} \mathrm{C}$ and the reaction mixture was stirred next at $-20^{\circ} \mathrm{C}$ for $12 \mathrm{~h}$. After addition of a few drops of $\mathrm{Et}_{3} \mathrm{~N}$, the solvent was removed in vacuo. The product was purified by flash chromatography (silylated $\mathrm{SiO}_{2}, 0.5 \%$ EtOAc in hexanes) to provide (+)-32 (14 mg, 26\% yield) as a yellow oil: $[\alpha]_{D}^{23}+34.6\left(c\right.$ 1.85, $C_{6} D_{6}$ ); IR (film) 2956 (s), 2930 (s), $2858(m), 2360$ (w), $1746(s)$, 1464 (s), 1398 (s), 1374 (m), 1279 (m), 1250 (s), 1073 (s), 1003 (s); ${ }^{1} \mathrm{H}$ NMR (500 MHz, $\mathrm{C}_{6} \mathrm{D}_{6}$ ) $\delta$ 7.02 (s, 1 H), $5.61(\mathrm{~m}, 1 \mathrm{H}), 5.02(\mathrm{~m}, 2 \mathrm{H}), 4.91$ (br s, $1 \mathrm{H}), 4.88$ (br s, $1 \mathrm{H}), 3.97(\mathrm{~m}, 1 \mathrm{H}), 3.74(\mathrm{~s}$, 3 H), 3.33 (s, 3 H), $2.91(m, 1 H), 2.71(m, 1 H), 2.22(s, 3 H), 2.21(m, 1 H)$, 2.16-2.02 (m, 2 H), 1.87-1.65 (m, 3 H), 1.75 (s, 3 H), 1.53 (d, J = 7.3 Hz, 3 H); $1.42(\mathrm{~d}, J=6.8 \mathrm{~Hz}, 3 \mathrm{H}), 1.55-1.33$ (m, $3 \mathrm{H}), 1.28(\mathrm{~m}, 1 \mathrm{H}), 1.15(\mathrm{~s}, 9 \mathrm{H}), 1.03(\mathrm{~d}, \mathrm{~J}=6.5 \mathrm{~Hz}, 3 \mathrm{H}), 0.98(\mathrm{~d}, \mathrm{~J}=6.7 \mathrm{~Hz}, 3 \mathrm{H}), 0.30(\mathrm{~s}, 6 \mathrm{H})$; ${ }^{13} \mathrm{C}$ NMR $\left(125 \mathrm{MHz}, \mathrm{C}_{6} \mathrm{D}_{6}\right) \delta 171.3,157.4,150.2,145.1,144.6,144.2,128.7,127.1,114.6,113.6$, $112.4,104.0,98.0,78.8,76.3,60.3,60.2,46.3,45.3,40.2,38.2,32.7,29.7,29.3,26.7,25.9$, $22.7,21.5,20.7,20.4,19.2,12.4,10.3,-3.8,-3.9$; high resolution mass spectrum (ES+) $\mathrm{m} / \mathrm{z}$ $649.3918\left[(\mathrm{M}+\mathrm{Na})^{+}\right.$; calcd for $\left.\mathrm{C}_{37} \mathrm{H}_{58} \mathrm{NaO}_{6} \mathrm{Si}: 649.3900\right]$.

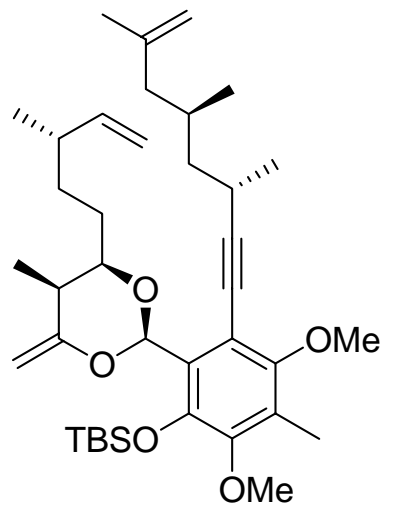


Enol-acetal (+)-33: To a solution of $(+)-32(53 \mathrm{mg}, 0.085 \mathrm{mmol})$ in THF (1 mL) was added $\mathrm{Cp}_{2} \mathrm{TiMe}_{2}(0.5 \mathrm{M}$ in hexanes, $0.254 \mathrm{mmol})$ and the mixture was heated at $60{ }^{\circ} \mathrm{C}$ for $12 \mathrm{~h}$. The reaction mixture was concentrated in vacuo and the product was purified by flash chromatography (silylated $\mathrm{SiO}_{2}$, hexanes/EtOAc 4:1) to yield (+)-33 (46 mg, 87\% yield) as a yellow oil: $[\alpha]_{D}^{23}+57.9\left(c 0.72, \mathrm{C}_{6} \mathrm{H}_{6}\right)$; IR (film) $2958(\mathrm{~s}), 2930(\mathrm{~s}), 2858(\mathrm{~m}), 1651(\mathrm{~m}), 1576(\mathrm{w})$, 1460 (s), 1395 (s), 1258 (s), 1078 (m), 999 (s), 840 (s); ${ }^{1} \mathrm{H}$ NMR (500 MHz, C $\left.6 \mathrm{D}_{6}\right) \delta 6.49$ (s, $1 \mathrm{H}$ ), 5.59 (ddd, J = 2.7, 9.2, $17.3 \mathrm{~Hz}, 1 \mathrm{H}$ ), 4.95 (m, 2 H), 4.83 (br s, 1 H), 4.80 (br s, 1 H), 4.66 (s, 1 H), $4.28(\mathrm{~s}, 1 \mathrm{H}), 3.94(\mathrm{~m}, 1 \mathrm{H}), 3.68(\mathrm{~s}, 3 \mathrm{H}), 3.26(\mathrm{~s}, 3 \mathrm{H}), 2.86(\mathrm{~m}, 1 \mathrm{H}), 2.19(\mathrm{~m}, 2 \mathrm{H}), 2.17(\mathrm{~s}, 3$ H), 2.04 (m, 2 H), 1.78 (m, 2 H), 1.75 (s, 3 H), $1.63(\mathrm{~m}, 1 \mathrm{H}), 1.59$ (d, J = 7.0 Hz, 3 H), 1.48-1.35 (m, $3 \mathrm{H}), 1.29(\mathrm{~d}, \mathrm{~J}=6.8 \mathrm{~Hz}, 3 \mathrm{H}), 1.21(\mathrm{~m}, 1 \mathrm{H}), 1.11(\mathrm{~s}, 9 \mathrm{H}), 0.94(\mathrm{~d}, \mathrm{~J}=6.6 \mathrm{~Hz}, 3 \mathrm{H}), 0.93(\mathrm{~d}, \mathrm{~J}$ $=6.7 \mathrm{~Hz}, 3 \mathrm{H}), 0.28(\mathrm{~s}, 3 \mathrm{H}), 0.25(\mathrm{~s}, 3 \mathrm{H}) ;{ }^{13} \mathrm{C} N M R\left(125 \mathrm{MHz}, \mathrm{C}_{6} \mathrm{D}_{6}\right) \delta 164.2,156.9,149.8$, $144.8,144.5,143.6,128.6,126.9,114.2,113.0,112.0,102.0,98.8,92.6,87.8,76.6,59.9,59.7$, $46.0,44.8,38.1,37.6,32.5,30.3,28.9,26.5,25.6,22.3,21.0,20.2,20.0,18.9,14.6,10.0,-4.1$, 4.2; high resolution mass spectrum (ES+) $\mathrm{m} / \mathrm{z} 647.4122\left[(\mathrm{M}+\mathrm{Na})^{+}\right.$; calcd for $\mathrm{C}_{38} \mathrm{H}_{60} \mathrm{NaO}_{5} \mathrm{Si}$ : 647.4107].

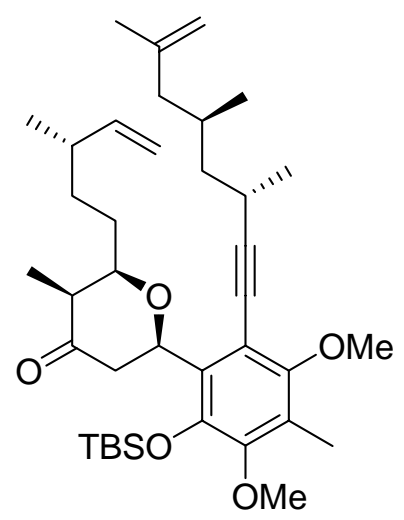

Pyranone (+)-34: A solution of (+)-33 (17 mg, $0.027 \mathrm{mmol})$ in $\mathrm{CH}_{2} \mathrm{Cl}_{2}(0.5 \mathrm{~mL})$ was treated with $\mathrm{Me}_{2} \mathrm{AlCl}(1 \mathrm{M}$ in hexanes, $0.033 \mathrm{mmol})$ at $-78{ }^{\circ} \mathrm{C}$ and then stirred for $20 \mathrm{~min}$ at $-78{ }^{\circ} \mathrm{C}$. The reaction mixture was quenched by addition of $\mathrm{Et}_{3} \mathrm{~N}(0.15 \mathrm{~mL})$ at $-78{ }^{\circ} \mathrm{C}$, followed by saturated aqueous $\mathrm{NaHCO}_{3}$ solution $(2 \mathrm{~mL})$. The mixture was extracted with $\mathrm{CH}_{2} \mathrm{Cl}_{2}(3 \times 10 \mathrm{~mL})$ and the combined organic layers were dried over $\mathrm{Na}_{2} \mathrm{SO}_{4}$ and concentrated in vacuo. Preparative thin- 
layer chromatography (hexanes/EtOAc 4:1) yielded (+)-34 (10.5 mg, 62\% yield) as a yellow oil: $[\alpha]_{\mathrm{D}}^{23}+40.9\left(c\right.$ 0.52, $\left.\mathrm{C}_{6} \mathrm{H}_{6}\right)$; IR (film) $2958(\mathrm{~s}), 2929(\mathrm{~s}), 2858(\mathrm{~m}), 1714(\mathrm{~s}), 1457(\mathrm{~s}), 1368(\mathrm{~m})$, $1259(\mathrm{~m}), 1073(\mathrm{~m}), 1004(\mathrm{~m}), 838(\mathrm{~s}) ;{ }^{1} \mathrm{H}$ NMR (500 MHz, $\left.\mathrm{C}_{6} \mathrm{D}_{6}\right) \delta 5.57$ (ddd, $\mathrm{J}=$ 2.9, 9.0, 17.1 Hz, 1 H), 5.51 (dd, J = 2.7, $12.1 \mathrm{~Hz}, 1 \mathrm{H}), 4.92$ (m, 2 H), 4.84 (br s, 1 H), 4.80 (br s, $1 \mathrm{H}), 4.09$ (dd, $J=12.3,14.2 \mathrm{~Hz}, 1 \mathrm{H}), 3.76(\mathrm{~m}, 1 \mathrm{H}), 3.65$ (s, $3 \mathrm{H}), 3.30(\mathrm{~s}, 3 \mathrm{H}), 2.76(\mathrm{~m}, 1 \mathrm{H}), 2.50-2.46$ (m, 2 H), 2.16 (s, 3 H), 2.1 (dd, J = 6.7, 13.2 Hz, 1 H), 1.97 (m, 2 H), 1.79 (m, 2 H), 1.67 (s, 3 H), 1.62-1.40 (m, $2 \mathrm{H}), 1.39(\mathrm{~d}, \mathrm{~J}=7.0 \mathrm{~Hz}, 3 \mathrm{H}), 1.48-1.35(\mathrm{~m}, 2 \mathrm{H}), 1.29(\mathrm{~d}, J=6.8 \mathrm{~Hz}, 3 \mathrm{H}), 1.21$ (m, 1 H), 1.07 (s, 9 H), 0.91 (d, J = 6.6 Hz, 3 H), 0.90 (d, J = 6.7 Hz, 3 H), $0.21(\mathrm{~s}, 3 \mathrm{H}), 0.20$ (s, 3 $\mathrm{H}) ;{ }^{13} \mathrm{C}$ NMR $\left(125 \mathrm{MHz}, \mathrm{C}_{6} \mathrm{D}_{6}\right) \delta 209.5,157.1,150.2,144.5,144.4,142.8,130.9,128.7,125.8$, 113.7, 113.0, 112.1, 103.6, 80.7, 76.8, 73.8, 59.9, 59.7, 49.7, 46.0, 44.7, 43.1, 38.1, 30.5, 28.9, $26.3,25.5,22.3,20.8,20.2,19.6,18.8,11.0,9.9,-3.9,-4.3$; high resolution mass spectrum (ES+) $\mathrm{m} / \mathrm{z} 647.4079\left[(\mathrm{M}+\mathrm{Na})^{+}\right.$; calcd for $\left.\mathrm{C}_{38} \mathrm{H}_{60} \mathrm{NaO}_{5} \mathrm{Si}: 647.4108\right]$.

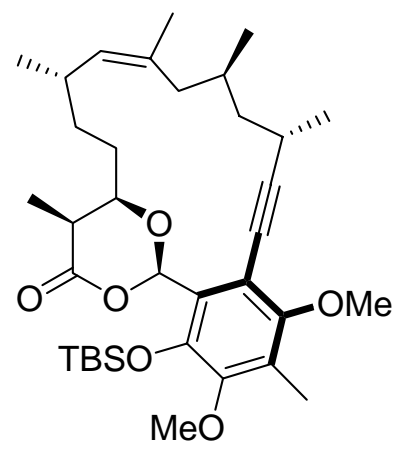

$Z$ Olefin (+)-36: To a solution of $(+)-32(30 \mathrm{mg}, 0.048 \mathrm{mmol})$ in $\mathrm{CH}_{2} \mathrm{Cl}_{2}(15 \mathrm{~mL})$ was added Grubbs' second generation catalyst (12 $\mathrm{mg}, 30 \mathrm{~mol} \%)$ and the reaction mixture was heated at reflux for $45 \mathrm{~h}$. Two additional portions of catalyst ( $2 \times 8 \mathrm{mg}, 20 \mathrm{~mol} \%$ each) were added during the reaction. The solvent was removed in vacuo and the product was purified twice by flash chromatography (silylated $\mathrm{SiO}_{2}$, hexanes/EtOAc 24:1) to provide (+)-36 (10 mg, 35\% yield) as a yellow oil: $[\alpha]_{D}^{23}+43.7\left(c \quad 0.400, \mathrm{CH}_{2} \mathrm{Cl}_{2}\right.$ ); IR (film) 2957 (s), 2929 (s), 2857 (m), 1763 (s), 1728 (m), 1462 (s), 1392 (s), $1279(\mathrm{~m}), 1251(\mathrm{~m}), 1206(\mathrm{~m}), 1068(\mathrm{~m}), 1002(\mathrm{~m}), 837$ (s); ${ }^{1} \mathrm{H}$ NMR (500 $\left.\mathrm{MHz}, \mathrm{C}_{6} \mathrm{D}_{6}\right) \delta 7.32(\mathrm{~s}, 1 \mathrm{H}), 5.06(\mathrm{~d}, \mathrm{~J}=10.0 \mathrm{~Hz}, 1 \mathrm{H}), 4.10(\mathrm{~m}, 1 \mathrm{H}), 3.63(\mathrm{~s}, 3 \mathrm{H}), 3.30(\mathrm{~s}, 3 \mathrm{H})$, $3.11(m, 1 \mathrm{H}), 2.73(\mathrm{~m}, 1 \mathrm{H}), 2.48(\mathrm{~d}, \mathrm{~J}=13.2 \mathrm{~Hz}, 1 \mathrm{H}), 2.37(\mathrm{~m}, 1 \mathrm{H}), 2.15(\mathrm{~s}, 3 \mathrm{H}), 2.08(\mathrm{~m}, 1 \mathrm{H})$, 
$1.88(\mathrm{~m}, 1 \mathrm{H}), 1.72(\mathrm{~s}, 3 \mathrm{H}), 1.49-1.30(\mathrm{~m}, 5 \mathrm{H}), 1.29(\mathrm{~d}, \mathrm{~J}=6.8 \mathrm{~Hz}, 3 \mathrm{H}), 1.10(\mathrm{~d}, \mathrm{~J}=6.8 \mathrm{~Hz}, 3$ H), $1.03(\mathrm{~s}, 9 \mathrm{H}), 0.98(\mathrm{~d}, J=6.5 \mathrm{~Hz}, 3 \mathrm{H}), 0.95(\mathrm{~d}, J=6.5 \mathrm{~Hz}, 3 \mathrm{H}), 0.93(\mathrm{~m}, 1 \mathrm{H}), 0.19(\mathrm{~s}, 6 \mathrm{H})$;

${ }^{13} \mathrm{C}$ NMR $\left(125 \mathrm{MHz}, \mathrm{C}_{6} \mathrm{D}_{6}\right) \delta 171.9,157.2,150.1,143.9,133.8,133.6,128.7,126.6,115.2,104.0$, $93.7,77.0,76.7,60.3,46.7,39.6,38.6,34.2,33.4,33.1,32.6,30.6,26.6,26.3,25.7,23.5,22.0$, 20.9, 19.2, 11.2, 10.3, -3.9, -4.1; high resolution mass spectrum (ES+) $\mathrm{m} / \mathrm{z} 621.3576\left[(\mathrm{M}+\mathrm{Na})^{+}\right.$; calcd for $\left.\mathrm{C}_{35} \mathrm{H}_{54} \mathrm{NaO}_{6} \mathrm{Si}: 621.3587\right]$.

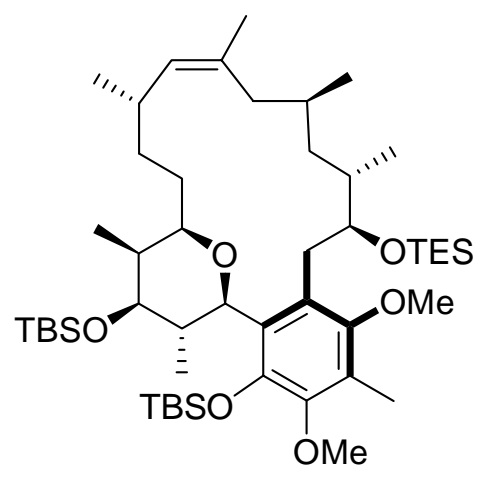

C(19)-TES ether (-)-39: To a solution of (+)-30 (43 mg, $0.058 \mathrm{mmol})$ in pyridine $(0.5 \mathrm{~mL})$ at $0{ }^{\circ} \mathrm{C}$ was added DMAP (14 mg, $0.116 \mathrm{mmol})$, TESOTf $(65 \mu \mathrm{L}, 0.288 \mathrm{mmol})$, and 2,6-lutidine $(67 \mu \mathrm{L}$, $0.576 \mathrm{mmol})$, then the reaction mixture was allowed to reach room temperature and stirred for 1 h. The reaction was quenched with saturated aqueous $\mathrm{NaHCO}_{3}(0.5 \mathrm{~mL})$, and extracted with $\mathrm{Et}_{2} \mathrm{O}$ $(3 \times 10 \mathrm{~mL})$. The combined organic extracts were dried over $\mathrm{Na}_{2} \mathrm{SO}_{4}$, and then concentrated in vacuo. Preparative thin-layer chromatography purification with $3 \%$ ethyl acetate in hexanes as eluent afforded (-)-39 (44 mg, 89\% yield) as a colorless oil: $[\alpha]_{\mathrm{D}}^{23}-20.3\left(\mathrm{c} 0.630, \mathrm{CHCl}_{3}\right)$; IR $\left(\mathrm{CHCl}_{3}\right) 2950$ (s), 1457 (s), 1407 (m), 1376 (s), 1253 (s), 1106 (s), 1068 (s), 1006 (s), 883 (m), 837 (s), $775(\mathrm{~m}) \mathrm{cm}^{-1} ;{ }^{1} \mathrm{H}$ NMR $\left(500 \mathrm{MHz}, \mathrm{C}_{6} \mathrm{D}_{6}\right) \delta 5.20(\mathrm{~d}, \mathrm{~J}=8.9 \mathrm{~Hz}, 1 \mathrm{H}), 5.06(\mathrm{~d}, \mathrm{~J}=10.4 \mathrm{~Hz}$, $1 \mathrm{H}), 4.66(\mathrm{dd}, J=2.1,7.5 \mathrm{~Hz}, 1 \mathrm{H}), 3.99(\mathrm{dd}, \mathrm{J}=9.4,14.7 \mathrm{~Hz}, 1 \mathrm{H}), 3.87(\mathrm{~d}, J=8.5 \mathrm{~Hz}, 1 \mathrm{H})$, 3.72 (dd, J = 4.6, $10.1 \mathrm{~Hz}, 1 \mathrm{H}), 3.47$ (s, $3 \mathrm{H}$ ), 3.14 (s, $3 \mathrm{H}), 2.70$ (d, J = $14.3 \mathrm{~Hz}, 1 \mathrm{H}), 2.62$ (s, 1 H), $2.55(\mathrm{~m}, 1 \mathrm{H}), 2.39$ (apparent t, J = $4.8 \mathrm{~Hz}, 1 \mathrm{H}), 2.24(\mathrm{~s}, 3 \mathrm{H}), 2.19(\mathrm{~m}, 1 \mathrm{H}), 2.08(\mathrm{~m}, 1 \mathrm{H})$, $1.91(\mathrm{~d}, \mathrm{~J}=10.7 \mathrm{~Hz}, 1 \mathrm{H}), 1.87(\mathrm{~m}, 1 \mathrm{H}), 1.80(\mathrm{~m}, 1 \mathrm{H}), 1.76(\mathrm{~s}, 3 \mathrm{H}), 1.67$ (apparent t, $\mathrm{J}=13.0$ Hz, 1 H), 1.55 (apparent t, J = 13.0 Hz, 1 H), $1.46(d, J=7.0$ Hz, 3 H), 1.40-1.31 (m, 3 H), 1.12 (s, 9 H), $1.07(\mathrm{~s}, 9 \mathrm{H}), 1.06(\mathrm{~d}, \mathrm{~J}=7.2 \mathrm{~Hz}, 3 \mathrm{H}$ ), $0.99(\mathrm{~d}, \mathrm{~J}=7.5 \mathrm{~Hz}, 3 \mathrm{H}$ ), 0.98 (apparent t, $\mathrm{J}=7.9$ 
$\mathrm{Hz}, 9 \mathrm{H}), 0.97(\mathrm{~d}, J=6.5 \mathrm{~Hz}, 3 \mathrm{H}), 0.96(\mathrm{~d}, J=7.6 \mathrm{~Hz}, 3 \mathrm{H}), 0.71-0.53(\mathrm{~m}, 6 \mathrm{H}), 0.33(\mathrm{~s}, 3 \mathrm{H})$, 0.20 (s, $3 \mathrm{H}), 0.19$ (s, $3 \mathrm{H}), 0.02$ (s, $3 \mathrm{H}) ;{ }^{13} \mathrm{C}$ NMR (125 MHz, $\left.\mathrm{C}_{6} \mathrm{D}_{6}\right) \delta$ 154.1, 148.5, 143.0, 135.1, $132.3,130.7,129.7,123.7,79.4,79.3,77.5,77.2,59.3,58.8,43.2,41.6,38.3,37.9,36.8,32.8$, $31.1,30.6,30.5,27.9,26.4,26.1,23.6,21.3,19.7,18.8,18.4,17.2,14.3,9.9,8.4,7.2,5.2,-3.7$, $3.9,-4.5,-4.9$; high resolution mass spectrum $(\mathrm{ES}+) \mathrm{m} / \mathrm{z} 883.6021\left[(\mathrm{M}+\mathrm{Na})^{+}\right.$; calcd for $\left.\mathrm{C}_{49} \mathrm{H}_{92} \mathrm{O}_{6} \mathrm{Si}_{3} \mathrm{Na}: 883.6098\right]$.

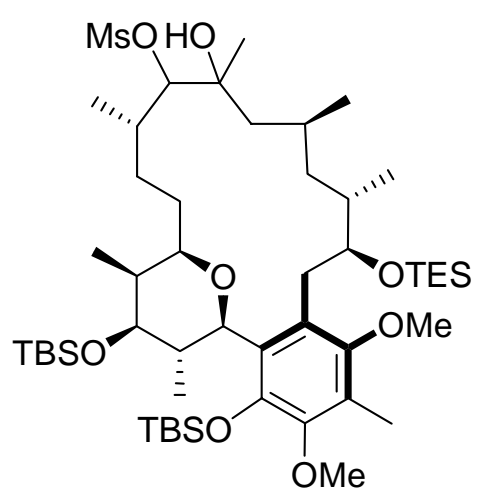

Monomesylate (+)-40: A solution of $(-)-39(84 \mathrm{mg}, 0.098 \mathrm{mmol})$ and pyridine $(0.3 \mathrm{~mL})$ in THF (2 $\mathrm{mL}$ ) was treated with $\mathrm{OsO}_{4}(28 \mathrm{mg}, 0.11 \mathrm{mmol})$ at $0{ }^{\circ} \mathrm{C}$, and then the reaction mixture was stirred for $5 \mathrm{~h}$ at the same temperature. The reaction mixture was quenched with a 1:1 (v/v) mixture of saturated aqueous $\mathrm{NaHSO}_{3}$ and saturated aqueous $\mathrm{NaHCO}_{3}(1 \mathrm{~mL})$, and $\mathrm{MeOH}(0.5 \mathrm{~mL})$, allowed to reach room temperature, stirred for an additional $1 \mathrm{~h}$, and then extracted with $\mathrm{Et}_{2} \mathrm{O}(3 \mathrm{x}$ $25 \mathrm{~mL}$ ). The combined organic extracts were washed with saturated aqueous $\mathrm{NaHCO}_{3}$ and brine (15 $\mathrm{mL}$ each), dried over $\mathrm{Na}_{2} \mathrm{SO}_{4}$, and then concentrated in vacuo. Preparative thin-layer chromatography purification with ethyl acetate-hexanes $(1: 4)$ as eluent afforded the $C(13,14)$ diol (69 mg, 78\% yield) as a colorless oil: $[\alpha]_{\mathrm{D}}^{23}+35.3\left(\mathrm{c} \mathrm{1.780}, \mathrm{C}_{6} \mathrm{H}_{6}\right)$; IR $\left(\mathrm{CHCl}_{3}\right) 3386(\mathrm{br}), 2950(\mathrm{~s})$, 1458 (s), 1380 (s), 1253 (s), 1068 (s), 1006 (s), 883 (m), 840 (s), 779 (m) cm ${ }^{-1} ;{ }^{1} \mathrm{H}$ NMR (500 $\left.\mathrm{MHz}, \mathrm{C}_{6} \mathrm{D}_{6}\right) \delta 4.92(\mathrm{~d}, \mathrm{~J}=10.5 \mathrm{~Hz}, 1 \mathrm{H}$ ), 4.47 (apparent quintet, $\mathrm{J}=3.5 \mathrm{~Hz}, 1 \mathrm{H}), 3.65-3.54(\mathrm{~m}, 3$ H), 3.43 (s, 3 H), 3.42 (s, 3 H), 3.14 (apparent t, J = 5.7 Hz, 1 H), 2.98 (dd, J = 3.8, 13.2 Hz, 1 H), 2.22 (apparent d, J = 4.4 Hz, $1 \mathrm{H}), 2.17(\mathrm{~s}, 3 \mathrm{H}), 2.15(\mathrm{~m}, 1 \mathrm{H}), 1.99(\mathrm{~m}, 1 \mathrm{H}), 1.90-1.83(\mathrm{~m}, 2 \mathrm{H})$, $1.80(\mathrm{dd}, \mathrm{J}=2.1,14.5 \mathrm{~Hz}, 1 \mathrm{H})$, 1.78-1.67 (m, $3 \mathrm{H}), 1.59$ (m, $1 \mathrm{H}), 1.54-1.46$ (m, $2 \mathrm{H}), 1.35$ (m, 1 
H), $2.55(\mathrm{~d}, \mathrm{~J}=6.9 \mathrm{~Hz}, 3 \mathrm{H}), 1.24(\mathrm{~s}, 3 \mathrm{H}), 2.32(\mathrm{~d}, \mathrm{~J}=6.5 \mathrm{~Hz}, 3 \mathrm{H}), 1.12$ (s, $9 \mathrm{H}), 1.11$ (apparent t, $J=7.9 \mathrm{~Hz}, 9 \mathrm{H}), 1.05(\mathrm{~d}, J=6.8 \mathrm{~Hz}, 3 \mathrm{H}), 0.98(\mathrm{~s}, 9 \mathrm{H}), 0.96(\mathrm{~d}, J=6.4 \mathrm{~Hz}, 3 \mathrm{H}), 0.78$ (apparent q, $J=7.9 \mathrm{~Hz}, 6 \mathrm{H}), 0.71(\mathrm{~d}, \mathrm{~J}=6.6 \mathrm{~Hz}, 3 \mathrm{H}), 0.31(\mathrm{~s}, 3 \mathrm{H}), 0.21(\mathrm{~s}, 3 \mathrm{H}), 0.10(\mathrm{~s}, 3 \mathrm{H})$, 0.09 (s, $3 \mathrm{H}) ;{ }^{13} \mathrm{C}$ NMR $\left(125 \mathrm{MHz}, \mathrm{C}_{6} \mathrm{D}_{6}\right) \delta 154.6,148.9,143.5,129.6,129.5,124.4,82.6,81.1$, $79.3,79.2,78.8,75.5,59.8,59.4,42.7,42.6,41,8,39.1,37.5,36.9,35.4,32.9,32.0,28.5,26.9$, $26.5,26.0,22.4,18.7,18.3,17.6,16.9,13.6,10.0,7.7,7.6,5.9,-3.8,-4.0,-4.4,-4.7$; high resolution mass spectrum (ES+) $\mathrm{m} / z$ 917.6132 [(M+Na) ${ }^{+}$; calcd for $\mathrm{C}_{49} \mathrm{H}_{94} \mathrm{O}_{8} \mathrm{Si}_{3} \mathrm{Na}$ : 917.6154].

To a solution of the above diol $(56 \mathrm{mg}, 0.063 \mathrm{mmol})$ and pyridine $(0.5 \mathrm{~mL})$ in dichloromethane $(1.5 \mathrm{~mL})$ at $0{ }^{\circ} \mathrm{C}$ was added $\mathrm{MsCl}(98 \mu \mathrm{L}, 1.26 \mathrm{mmol})$, and then the reaction mixture was stirred at $0{ }^{\circ} \mathrm{C}$ for $2 \mathrm{~h}$. The reaction was quenched with saturated aqueous $\mathrm{NaHCO}_{3}(0.5 \mathrm{~mL})$ and extracted with $\mathrm{Et}_{2} \mathrm{O}(3 \times 25 \mathrm{~mL})$. The combined organic extracts were washed with brine $(15 \mathrm{~mL})$, dried over $\mathrm{Na}_{2} \mathrm{SO}_{4}$, and then concentrated in vacuo. Preparative thin-layer chromatography purification with $30 \%$ ethyl acetate in hexanes as eluent afforded (+)-40 (58 mg, 95\% yield) as a colorless oil: $[\alpha]_{\mathrm{D}}^{23}+21.1\left(c\right.$ 1.880, $\left.\mathrm{C}_{6} \mathrm{H}_{6}\right)$; IR $\left(\mathrm{CHCl}_{3}\right) 3532(\mathrm{br}), 2950$ (s), 2275 (w), 1735 (w), 1457 (s), 1338 (s), 1253 (s), 1172 (m), 1068 (s), 1006 (s), 921 (m), 840 (s), 779 (m) cm ${ }^{-1} ;{ }^{1} \mathrm{H}$ NMR (500 MHz, $\left.\mathrm{C}_{6} \mathrm{D}_{6}\right) \delta 4.97(\mathrm{~d}, J=10.4 \mathrm{~Hz}, 1 \mathrm{H}), 4.68(\mathrm{~d}, \mathrm{~J}=2.9 \mathrm{~Hz}, 1 \mathrm{H}), 4.67(\mathrm{~m}, 1 \mathrm{H}), 3.63-3.55(\mathrm{~m}, 3 \mathrm{H})$, 3.45 (s, 3 H), 3.41 (s, 3 H), 3.18 (dd, J = 4.3, 13.5 Hz, 1 H), $2.29(\mathrm{~s}, 3 \mathrm{H}), 2.26$ (m, $1 \mathrm{H}), 2.20$ (s, 3 H), 2.21-2.06 (m, 2 H), $1.94(m, 1$ H), 1.90 (dd, J = 2.5, 14.4 Hz, 1 H), $1.83(m, 1$ H), 1.70-1.40 (m, $6 \mathrm{H}), 1.31(\mathrm{~d}, \mathrm{~J}=7.0 \mathrm{~Hz}, 3 \mathrm{H}), 1.28(\mathrm{~m}, 1 \mathrm{H}), 1.24(\mathrm{~s}, 3 \mathrm{H}), 2.21(\mathrm{~d}, \mathrm{~J}=6.7 \mathrm{~Hz}, 3 \mathrm{H}), 1.14(\mathrm{~s}, 9$ H), 1.09 (apparent t, J = 7.9 Hz, 9 H), 1.02 (m, 1 H), 1.00 (s, 9 H), 0.99 (d, J = 6.8 Hz, 3 H), 0.87 $(\mathrm{d}, \mathrm{J}=6.2 \mathrm{~Hz}, 3 \mathrm{H}), 0.78$ (d, J = 6.5 Hz, $3 \mathrm{H}), 0.74(\mathrm{~m}, 6 \mathrm{H}), 0.32(\mathrm{~s}, 3 \mathrm{H}), 0.28(\mathrm{~s}, 3 \mathrm{H}), 0.13$ (s, 6 H); ${ }^{13} \mathrm{C}$ NMR $\left(125 \mathrm{MHz}, \mathrm{C}_{6} \mathrm{D}_{6}\right) \delta$ 154.4, 148.9, 143.5, 129.7, 129.4, 124.3, 91.4, 79.9, 79.3, 79.1, $77.5,75.9,59.8,59.3,47.4,41.9,40.3,38.8,38.0,37.9,34.6,34.2,33.0,32.2,27.9,26.6,26.4$, 26.0, 22.1, 18.7, 18.4, 16.5, 16.4, 13.6, 9.9, 7.7, 7.4, 5.7, -3.7, -4.0, -4.5, -4.7; high resolution mass spectrum (ES+) $\mathrm{m} / \mathrm{z} 995.5943\left[(\mathrm{M}+\mathrm{Na})^{+}\right.$; calcd for $\mathrm{C}_{50} \mathrm{H}_{96} \mathrm{O}_{10} \mathrm{SSi}_{3} \mathrm{Na}$ : 995.5929]. 


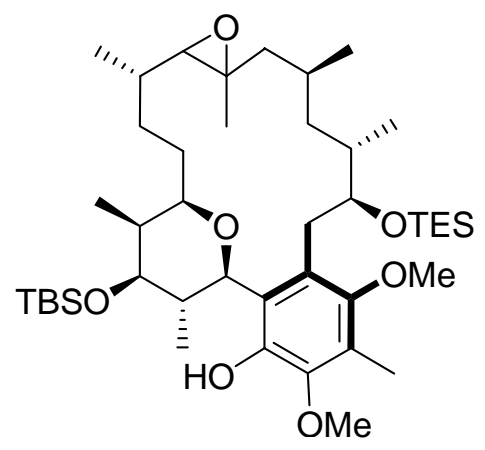

Trans epoxide (+)-41: To a solution of (+)-40 (69 mg, $0.071 \mathrm{mmol})$ in THF (2 mL) was added 40 $\% \mathrm{BnNMe}_{3} \mathrm{OH}$ in $\mathrm{MeOH}(63 \mu \mathrm{L}, 0.142 \mathrm{mmol})$ at $0{ }^{\circ} \mathrm{C}$, and then the reaction mixture was stirred at $0{ }^{\circ} \mathrm{C}$ for $1 \mathrm{~h}$. The reaction mixture was then concentrated and directly purified by preparative thinlayer chromatography with ethyl acetate-hexanes (1:4) as eluent, to afford (+)-41 (45 mg, 84\% yield) as a colorless oil: $[\alpha]_{\mathrm{D}}^{23}+65.8\left(\mathrm{c} 0.975, \mathrm{C}_{6} \mathrm{H}_{6}\right)$; IR $\left(\mathrm{CHCl}_{3}\right) 3340(\mathrm{br}), 2931(\mathrm{~s}), 1697(\mathrm{w})$, 1458 (s), 1376 (m), 1253 (m), 1079 (s), 841 (s), 736 (w) cm ${ }^{-1} ;{ }^{1} \mathrm{H}$ NMR (500 MHz, $\left.\mathrm{C}_{6} \mathrm{D}_{6}\right) \delta 8.28$ (s, $1 \mathrm{H}), 4.69(\mathrm{~d}, J=10.5 \mathrm{~Hz}, 1 \mathrm{H}), 3.99(\mathrm{~m}, 1 \mathrm{H}), 3.93(\mathrm{~s}, 3 \mathrm{H}), 3.69(\mathrm{~m}, 1 \mathrm{H}), 3.67(\mathrm{dd}, J=4.5,10.3$ Hz, 1 H), 3.32 (m, 1 H), 3.31 (s, 3 H), 2.79 (dd, J = 4.3, 13.2 Hz, 1 H), $2.70(d, J=6.1$ Hz, 1 H), $2.47(\mathrm{~m}, 1 \mathrm{H}), 2.28(\mathrm{~s}, 3 \mathrm{H}), 1.82(\mathrm{~m}, 1 \mathrm{H}), 1.75-1.53(\mathrm{~m}, 5 \mathrm{H}), 1.47(\mathrm{~m}, 1 \mathrm{H}), 1.37(\mathrm{~m}, 1 \mathrm{H}), 1.26$ (d, J = 6.6 Hz, $3 \mathrm{H}$ ), 1.15 (s, $3 \mathrm{H}$ ), 1.14 (apparent t, $J=8.0 \mathrm{~Hz}, 9 \mathrm{H}), 1.13(\mathrm{~d}, \mathrm{~J}=6.3 \mathrm{~Hz}, 3 \mathrm{H}$ ), $0.98(\mathrm{~s}, 9 \mathrm{H}), 0.92(\mathrm{~d}, \mathrm{~J}=6.7 \mathrm{~Hz}, 3 \mathrm{H}), 0.90(\mathrm{~d}, \mathrm{~J}=7.1 \mathrm{~Hz}, 3 \mathrm{H}), 0.80(\mathrm{~m}, 6 \mathrm{H}), 0.77(\mathrm{~d}, \mathrm{~J}=6.7$ $\mathrm{Hz}, 3 \mathrm{H}), 0.72(\mathrm{~m}, 1 \mathrm{H}), 0.13(\mathrm{~s}, 6 \mathrm{H}), 0.09(\mathrm{~s}, 3 \mathrm{H}) ;{ }^{13} \mathrm{C}$ NMR $\left(125 \mathrm{MHz}, \mathrm{C}_{6} \mathrm{D}_{6}\right) \delta$ 151.0, 147.5, $146.9,124.9,123.7,123.0,83.2,82.7,78.3,74.3,65.8,60.2,59.9,59.3,45.2,42.3,42.0,37.1$, $34.9,34.7,33.9,33.8,31.7,31.5,28.5,25.9,21.5,19.7,18.3,15.3,14.4,10.1,7.4,6.7,6.1,-4.1$, -4.8; high resolution mass spectrum (ES+) $\mathrm{m} / \mathrm{z} 785.5213\left[(\mathrm{M}+\mathrm{Na})^{+}\right.$; calcd for $\mathrm{C}_{43} \mathrm{H}_{78} \mathrm{O}_{7} \mathrm{Si}_{2} \mathrm{Na}$ : 785.5138]. 


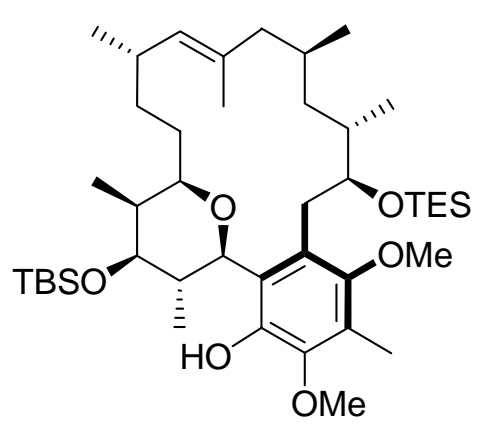

E Olefin (-)-42: $n$-BuLi (2.5M in hexanes, $0.4 \mathrm{~mL}, 1.0 \mathrm{mmol}$ ) was added to a solution of $\mathrm{WCl}_{6}$ (198 $\mathrm{mg}, 0.5 \mathrm{mmol}$ ) in THF $(5 \mathrm{~mL})$, at $-78{ }^{\circ} \mathrm{C}$, and then the reaction mixture was allowed to warm to room temperature and stirred for $20 \mathrm{~min}$. A solution of (+)-41 (44 mg, $0.058 \mathrm{mmol})$ in THF (0.6 $\mathrm{mL}$ ) was treated next with the $\left[\mathrm{W}^{4+}\right]$ solution described above $(1.25 \mathrm{~mL})$, at $0{ }^{\circ} \mathrm{C}$, and then the reaction mixture was allowed to warm to room temperature and stirred for an additional 40 min. The reaction was quenched at $0{ }^{\circ} \mathrm{C}$ with saturated aqueous $\mathrm{NaHCO}_{3}(1 \mathrm{~mL})$, and the mixture was extracted with $\mathrm{Et}_{2} \mathrm{O}(3 \times 25 \mathrm{~mL})$. The combined organic layers were dried over $\mathrm{Na}_{2} \mathrm{SO}_{4}$, and then concentrated in vacuo. Preparative thin-layer chromatography with $15 \%$ ethyl acetate in hexanes as eluent afforded (-)-42 (31 mg, 71\% yield) as a colorless oil, contaminated with $10-12 \%$ of an unidentified isomer. (-)-42: $[\alpha]_{\mathrm{D}}^{23}-310.4\left(c\right.$ 0.710, $\left.\mathrm{C}_{6} \mathrm{H}_{6}\right)$; IR $\left(\mathrm{CHCl}_{3}\right) 2953(\mathrm{~s}), 2360(w), 1739(w)$, 1458 (m), 1378 (m), 1254 (m), 1109 (s), 1074 (s), 1010 (s), 839 (m); ${ }^{1} \mathrm{H} \mathrm{NMR}^{8}$ (500 MHz, $\left.\mathrm{C}_{6} \mathrm{D}_{6}\right) \delta$ $4.94(d, J=9.5 \mathrm{~Hz}, 1 \mathrm{H}), 4.60$ (d, J = 10.5 Hz, $1 \mathrm{H}$ ), 4.17 (dd, J = 5.0, $10.8 \mathrm{~Hz}, 1 \mathrm{H}$ ), 3.73 (dd, $J=$ 4.6, 10.0 Hz, 1 H), $3.62(m, 1$ H), 3.58 (s, 3 H), 3.50 (m, 1 H), 3.35 (s, 3 H), $2.85(m, 1$ H), 2.83 (dd, J = 5.1, $13.7 \mathrm{~Hz}, 1 \mathrm{H}), 2.29(\mathrm{~m}, 1 \mathrm{H}), 2.21(\mathrm{~s}, 3 \mathrm{H}), 2.00(\mathrm{~m}, 1 \mathrm{H}), 1.89(\mathrm{~m}, 1 \mathrm{H}), 1.86(\mathrm{br} d, J$ $=14.7 \mathrm{~Hz}, 1 \mathrm{H}), 1.72-1.49(\mathrm{~m}, 5 \mathrm{H}), 1.47(\mathrm{~s}, 3 \mathrm{H}), 1.37-1.26(\mathrm{~m}, 2 \mathrm{H}), 1.28(\mathrm{~d}, \mathrm{~J}=7.0 \mathrm{~Hz}, 3 \mathrm{H})$, $1.26(\mathrm{~d}, \mathrm{~J}=6.5 \mathrm{~Hz}, 3 \mathrm{H}), 1.17$ (apparent t, J = 7.9 Hz, $9 \mathrm{H}$ ), $1.09(\mathrm{~m}, 1 \mathrm{H}), 1.01$ (s, $9 \mathrm{H}), 0.97$ (d, J $=6.4 \mathrm{~Hz}, 3 \mathrm{H}), 0.96(\mathrm{~d}, \mathrm{~J}=6.6 \mathrm{~Hz}, 3 \mathrm{H}), 0.84$ (apparent q, $\mathrm{J}=7.9 \mathrm{~Hz}, 6 \mathrm{H}), 0.77(\mathrm{~d}, \mathrm{~J}=6.6 \mathrm{~Hz}, 3$ H), $0.15(\mathrm{~s}, 3 \mathrm{H}), 0.14(\mathrm{~s}, 3 \mathrm{H}) ;{ }^{13} \mathrm{C}$ NMR $\left(125 \mathrm{MHz}, \mathrm{C}_{6} \mathrm{D}_{6}\right) \delta$ 151.1, 146.4, 146.3, 132.7, 132.0, 125.5, 124.6, 123.1, 83.6, 82.5, 79.3, 76.2, 60.4, 59.8, 44.4, 41.5, 40.2, 36.2, 35.9, 34.3, 33.4, $31.8,31.0,30.1,28.6,26.0,22.1,19.9,19.4,18.3,14.5,10.2,7.7,6.8,6.2,-4.0,-4.7$; high resolution mass spectrum (ES+) $\mathrm{m} / \mathrm{z} 769.5246\left[(\mathrm{M}+\mathrm{Na})^{+}\right.$; calcd for $\mathrm{C}_{43} \mathrm{H}_{78} \mathrm{O}_{6} \mathrm{Si}_{2} \mathrm{Na}$ : 769.5234].

\footnotetext{
${ }^{8} \mathrm{~A}$ signal for the $\mathrm{C}(4)$ phenol was not observed in the ${ }^{1} \mathrm{H}$ NMR spectrum recorded in $\mathrm{C}_{6} \mathrm{D}_{6}$.
} 


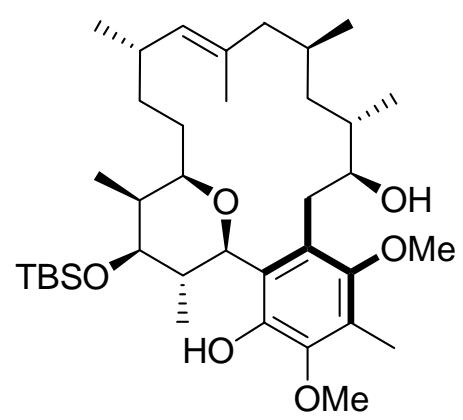

Alcohol (-)-43: A solution of (-)-42 (15 mg, $0.020 \mathrm{mmol})$ and pyridinium $p$-toluenesulfonate (1 $\mathrm{mg}, 0.004 \mathrm{mmol})$ in $\mathrm{MeOH}(1.5 \mathrm{~mL})$ was stirred for $6 \mathrm{~h}$ at $0{ }^{\circ} \mathrm{C}$. The reaction mixture was quenched with saturated aqueous $\mathrm{NaHCO}_{3}(0.5 \mathrm{~mL})$ and extracted with $\mathrm{Et}_{2} \mathrm{O}(3 \times 25 \mathrm{~mL})$. The combined organic extracts were washed with brine $(15 \mathrm{~mL})$, dried over $\mathrm{Na}_{2} \mathrm{SO}_{4}$, and then concentrated in vacuo. Preparative thin-layer chromatography purification with ethyl acetatehexanes (1:9) as eluent afforded (-)-43 (12 mg, 95\% yield) as a colorless oil: $[\alpha]_{D}^{23}-6.6(c$ 0.500, $\left.\mathrm{C}_{6} \mathrm{H}_{6}\right)$; IR $\left(\mathrm{CHCl}_{3}\right) 2930$ (s), 2857 (s), 1737 (w), 1458 (s), 1383 (m), 1255 (m), 1104 (s), 999 (m), 883 (w), $837(\mathrm{~m}) ;{ }^{1} \mathrm{H} \mathrm{NMR}^{9}\left(500 \mathrm{MHz}, \mathrm{C}_{6} \mathrm{D}_{6}\right) \delta 5.50(\mathrm{~s}, 1 \mathrm{H}), 5.12(\mathrm{~d}, J=10.8 \mathrm{~Hz}, 1 \mathrm{H}), 4.95(\mathrm{~d}, J$ $=9.7 \mathrm{~Hz}, 1 \mathrm{H}), 4.30(\mathrm{~m}, 1 \mathrm{H}), 3.62(\mathrm{dd}, \mathrm{J}=5.0,9.8 \mathrm{~Hz}, 1 \mathrm{H}), 3.61(\mathrm{~m}, 1 \mathrm{H}), 3.40(\mathrm{br} \mathrm{d}, J=10.9$ $\mathrm{Hz}, 1 \mathrm{H}$ ), $3.23(\mathrm{~s}, 3 \mathrm{H}), 3.12(\mathrm{dd}, \mathrm{J}=2.4,13.2 \mathrm{~Hz}, 1 \mathrm{H}), 3.02(\mathrm{~s}, 3 \mathrm{H}), 2.83(\mathrm{dd}, \mathrm{J}=5.1,13.1 \mathrm{~Hz}, 1$ H), $2.32(\mathrm{~m}, 1 \mathrm{H}), 2.24(\mathrm{~m}, 1 \mathrm{H}), 2.14(\mathrm{br} \mathrm{d}, \mathrm{J}=14.4 \mathrm{~Hz}, 1 \mathrm{H}), 1.97(\mathrm{~s}, 3 \mathrm{H}), 1.91(\mathrm{~m}, 1 \mathrm{H}), 1.89$ (m, $1 \mathrm{H}), 1.83-1.75$ (m, $2 \mathrm{H}), 1.74$ (dd, J = 8.7, $14.3 \mathrm{~Hz}, 1 \mathrm{H}), 1.65$ (s, $3 \mathrm{H}), 1.62$ (m, $1 \mathrm{H}), 1.49$ (m, $1 \mathrm{H}), 1.29(\mathrm{~d}, \mathrm{~J}=6.4 \mathrm{~Hz}, 3 \mathrm{H}), 1.24(\mathrm{~m}, 1 \mathrm{H}), 1.18(\mathrm{~d}, \mathrm{~J}=6.9 \mathrm{~Hz}, 3 \mathrm{H}), 1.17(\mathrm{~d}, \mathrm{~J}=6.7 \mathrm{~Hz}, 3$ H), 1.02 (s, 9 H), 1.01 (d, J = 6.6 Hz, 3 H), 0.95 (m, 1 H), 0.89 (d, J = 6.6 Hz, 3 H), $0.12(\mathrm{~s}, 3 \mathrm{H})$, $0.11(\mathrm{~s}, 3 \mathrm{H}) ;{ }^{13} \mathrm{C}$ NMR $\left(125 \mathrm{MHz}, \mathrm{C}_{6} \mathrm{D}_{6}\right) \delta 151.3,144.5,143.8,133.0,132.4,129.4,124.5,122.7$, $80.9,79.0,78.3,76.8,59.9,59.6,46.9,41.8,41.5,38.2,38.1,36.7,34.9,33.5,33.2,29.9,26.1$, 22.1, 21.9, 20.5, 18.3, 14.3, 13.3, 9.8, 7.4, -4.0, -4.6; high resolution mass spectrum (ES+) $\mathrm{m} / \mathrm{z}$ $655.4402\left[(\mathrm{M}+\mathrm{Na})^{+}\right.$; calcd for $\left.\mathrm{C}_{37} \mathrm{H}_{64} \mathrm{O}_{6} \mathrm{SiNa}: 655.4369\right]$.

\footnotetext{
${ }^{9} \mathrm{~A}$ signal for the $\mathrm{C}(4)$ phenol was not observed in the ${ }^{1} \mathrm{H}$ NMR spectrum recorded in $\mathrm{C}_{6} \mathrm{D}_{6}$.
} 


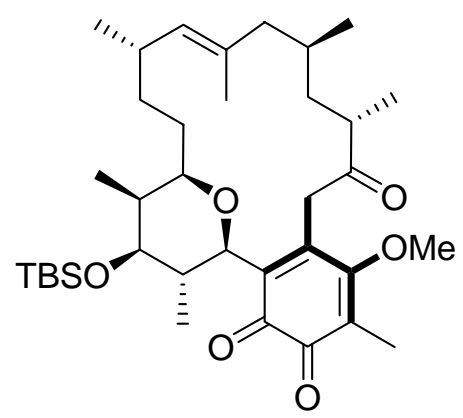

o-Quinone (-)-2: A solution of (-)-43 (12 mg, $0.019 \mathrm{mmol})$ and pyridine $(6 \mu \mathrm{L}, 0.072 \mathrm{mmol})$ in dichloromethane $(0.7 \mathrm{~mL})$ was treated with Dess-Martin periodinane $(32.2 \mathrm{mg}, 0.076 \mathrm{mmol})$ at 0 ${ }^{\circ} \mathrm{C}$, and then the reaction mixture was allowed to warm to room temperature and stirred for an additional $45 \mathrm{~min}$, in the dark. The reaction mixture was quenched with a 1:1 (v/v) mixture of saturated aqueous $\mathrm{Na}_{2} \mathrm{~S}_{2} \mathrm{O}_{3}$ and saturated aqueous $\mathrm{NaHCO}_{3}(0.5 \mathrm{~mL})$, and extracted with $\mathrm{Et}_{2} \mathrm{O}$ $(3 \times 5 \mathrm{~mL})$. The combined organic extracts were washed with brine $(2 \mathrm{~mL})$, dried over $\mathrm{Na}_{2} \mathrm{SO}_{4}$, and then concentrated in vacuo. Preparative thin-layer chromatography purification with ethyl acetate-hexanes (1:4) as eluent afforded (-)-2 (8 mg, 69\% yield) as a deep purple solid: m.p. 193-196 ${ }^{\circ} \mathrm{C} ;[\alpha]_{\mathrm{D}}^{23}-38.4(\mathrm{c} 0.320, \mathrm{MeCN}) ; \mathrm{IR}\left(\mathrm{CHCl}_{3}\right) 2924(\mathrm{~s}), 2358(\mathrm{w}), 1717(\mathrm{~m}), 1656(\mathrm{~m})$, $1584(\mathrm{~m}), 1516(\mathrm{~m}), 1456(\mathrm{~m}), 1378(\mathrm{~m}), 1328(\mathrm{~m}), 1254(\mathrm{~m}), 1083(\mathrm{~s}), 835(\mathrm{~m}) ;{ }^{1} \mathrm{H}$ NMR (500 $\left.\mathrm{MHz}, \mathrm{C}_{6} \mathrm{D}_{6}\right) \delta 4.79(\mathrm{~d}, \mathrm{~J}=9.9 \mathrm{~Hz}, 1 \mathrm{H}), 4.68(\mathrm{~d}, \mathrm{~J}=10.6 \mathrm{~Hz}, 1 \mathrm{H}), 4.24(\mathrm{~d}, \mathrm{~J}=17.2 \mathrm{~Hz}, 1 \mathrm{H}), 3.32$ (dd, J = 4.9, $9.9 \mathrm{~Hz}, 1 \mathrm{H}), 3.26$ (s, $3 \mathrm{H}), 3.20(\mathrm{br} \mathrm{d}, J=17.6 \mathrm{~Hz}, 1 \mathrm{H}), 3.11$ (d, J = $10.5 \mathrm{~Hz}, 1 \mathrm{H}$ ), $2.42(\mathrm{~m}, 1 \mathrm{H}), 2.12(\mathrm{~m}, 1 \mathrm{H}), 2.10(\mathrm{dd}, \mathrm{J}=4.0,13.4 \mathrm{~Hz}, 1 \mathrm{H}), 1.96(\mathrm{dd}, \mathrm{J}=4.1,13.4 \mathrm{~Hz}, 1 \mathrm{H})$, 1.82 (m, 1 H), 1.69 (s, 3 H), 1.68-1.53 (m, 4 H), 1.59 (s, 3 H), 1.40 (ddd, J = 3.5, 11.5, 13.8 Hz, 1 H), 1.37-1.16 (m, 2 H), $1.12(m, 1$ H), 1.01 (d, J = 7.5 Hz, 3 H), 1.00 (s, 9 H), 0.99 (d, J = 6.9 Hz, $3 \mathrm{H}), 0.90(\mathrm{~d}, \mathrm{~J}=6.5 \mathrm{~Hz}, 3 \mathrm{H}), 0.88(\mathrm{~d}, \mathrm{~J}=6.9 \mathrm{~Hz}, 3 \mathrm{H}), 0.76(\mathrm{~d}, \mathrm{~J}=6.5 \mathrm{~Hz}, 3 \mathrm{H}), 0.06(\mathrm{~s}, 3 \mathrm{H})$, 0.05 (s, $3 \mathrm{H}) ;{ }^{13} \mathrm{C}$ NMR $\left(125 \mathrm{MHz}, \mathrm{C}_{6} \mathrm{D}_{6}\right) \delta 180.1,179.2,166.1,161.1,147.2,136.8,135.7,129.8$, 120.6, 80.4, 77.6, 76.5, 61.6, 46.4, 42.6, 42.1, 41.3, 39.3, 38.9, 35.8, 33.8, 33.3, 28.7, 26.0, 22.0, 20.5, 18.7, 18.2, 15.2, 14.2, 9.9, 7.5, -4.1, -4.7; high resolution mass spectrum (ES+) $\mathrm{m} / \mathrm{z}$ $637.3886\left[(\mathrm{M}+\mathrm{Na})^{+}\right.$; calcd for $\left.\mathrm{C}_{36} \mathrm{H}_{58} \mathrm{O}_{6} \mathrm{SiNa}: 637.3900\right]$. 


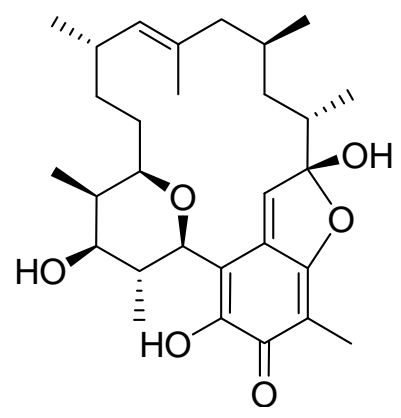

Synthetic (-)-Kendomycin (1): A solution of (-)-2 (3.5 mg, $5.7 \mu \mathrm{mol})$ in acetonitrile (1 mL) was treated with $48 \%$ aqueous $\mathrm{HF}(50 \mu \mathrm{L})$ at $0{ }^{\circ} \mathrm{C}$, in a plastic vial, and then the reaction mixture was allowed to warm to room temperature and stirred for an additional $20 \mathrm{~min}$, in the dark. A color change was observed from orange to yellow. The reaction mixture was quenched with saturated aqueous $\mathrm{NaHCO}_{3}(0.5 \mathrm{~mL})$, and extracted with EtOAc $(3 \times 5 \mathrm{~mL})$. The combined organic extracts were washed with saturated aqueous $\mathrm{NaHCO}_{3}$ and brine ( $3 \mathrm{~mL}$ each), dried over $\mathrm{Na}_{2} \mathrm{SO}_{4}$, and then concentrated in vacuo. Flash chromatography purification with ethyl acetate-hexanes (1:4 $\rightarrow$ 1:1) as eluent afforded (-)-1 (1.1 mg, 40\% yield) as a yellow solid: m.p. $235-238{ }^{\circ} \mathrm{C} ;[\alpha]_{\mathrm{D}}^{23}-79.3$ (c 0.135, MeOH), ${ }^{10} \mathrm{IR}\left(\mathrm{CHCl}_{3}\right) 3336$ (br), 2922 (s), 1667 (w), 1612 (s), 1574 (s), 1457 (m), 1378 (m), 1329 (s), 1252 (w), 1097 (s); ${ }^{1} \mathrm{H}$ NMR (500 MHz, Acetone-d $\left.d_{6}\right) \delta 8.05$ (br s, $\left.1 \mathrm{H}\right), 7.19$ (s, $1 \mathrm{H}$ ), $6.46(\mathrm{br} \mathrm{s}, 1 \mathrm{H}), 4.64(\mathrm{~d}, \mathrm{~J}=10.0 \mathrm{~Hz}, 1 \mathrm{H}), 4.35(\mathrm{~d}, \mathrm{~J}=10.4 \mathrm{~Hz}, 1 \mathrm{H}), 3.86(\mathrm{~d}, J=4.6 \mathrm{~Hz}, 1 \mathrm{H})$, $3.56(\mathrm{td}, \mathrm{J}=4.6,10.3 \mathrm{~Hz}, 1 \mathrm{H}), 3.53$ (ddd, $J=1.2,2.4,11.0 \mathrm{~Hz}, 1 \mathrm{H}), 2.41(\mathrm{~m}, 1 \mathrm{H}), 2.36(\mathrm{~m}, 1$ H), $2.12(\mathrm{br} \mathrm{d}, J=17.0 \mathrm{~Hz}, 1 \mathrm{H}), 1.96(\mathrm{~m}, 1 \mathrm{H}), 1.88(\mathrm{~m}, 1 \mathrm{H}), 1.84(\mathrm{~s}, 3 \mathrm{H}), 1.71(\mathrm{~m}, 1 \mathrm{H}), 1.67$ (m, 1 H), 1.63 (m, 1 H), 1.61 (s, 3 H), 1.57 (m, 2 H) 1.45 (ddd, J = 3.1, 11.1, 13.3 Hz, 1 H), 1.33 (m, $1 \mathrm{H}), 1.25(\mathrm{~m}, 1 \mathrm{H}), 0.95$ (d, J = $7.0 \mathrm{~Hz}, 3 \mathrm{H}), 0.94(\mathrm{~d}, J=6.5 \mathrm{~Hz}, 3 \mathrm{H}), 0.89(\mathrm{~d}, J=6.6 \mathrm{~Hz}, 3$ $\mathrm{H}), 0.87(\mathrm{~d}, \mathrm{~J}=6.6 \mathrm{~Hz}, 3 \mathrm{H}), 0.71(\mathrm{~d}, \mathrm{~J}=6.8 \mathrm{~Hz}, 3 \mathrm{H}) ;{ }^{13} \mathrm{C}$ NMR $\left(125 \mathrm{MHz}\right.$, Acetone- $\left.d_{6}\right) \delta 182.1$, $168.6,146.8,141.4,132.1,130.2,129.9,119.1,111.0,104.2,78.7,77.7,76.3,46.1,41.5,40.8$, $39.7,38.1,35.8,33.6,33.4,26.5,22.7,19.9,19.7,13.3,12.6,7.6,7.2$; high resolution mass spectrum (ES+) $m / z 487.3042\left[(\mathrm{M}+\mathrm{H})^{+}\right.$; calcd for $\left.\mathrm{C}_{29} \mathrm{H}_{43} \mathrm{O}_{6} \mathrm{Si}: 487.3059\right]$.

\footnotetext{
${ }^{10}$ Lit. values: m.p. $258^{\circ} \mathrm{C} ;[\alpha]_{D}^{20}-80.0$ (c 2.710, MeOH): Bode, H. B.; Zeeck, A. J. Chem. Soc., Perkin Trans. 1 2000, 323.
} 